\title{
Spiro-based thermally activated delayed fluorescence emitters with reduced non-radiative decay for high quantum efficiency, low roll-off, light-emitting diodes
}

\author{
Nidhi Sharma ${ }^{\ddagger a b}$, Michal Maciejczyk ${ }^{\ddagger c}$, David Hall ${ }^{\mathrm{b}, \mathrm{d}}$, Wenbo Li $^{\mathrm{a}}$, Vincent Liégeois ${ }^{\mathrm{f}}$, David \\ Beljonne $^{\mathrm{d} *}$, Yoann Olivier ${ }^{\mathrm{d}, \mathrm{e} *}$, Neil Robertson ${ }^{\mathrm{c} *}$, Ifor D. W. Samuel ${ }^{\mathrm{a} *}$ and Eli Zysman-Colman ${ }^{\mathrm{b} *}$ \\ ${ }^{a}$ Organic Semiconductor Centre, SUPA, School of Physics and Astronomy, University of St \\ Andrews, North Haugh, St Andrews, KY16 9SS, U.K._E-mail: idws@st-andrews.ac.uk \\ ${ }^{\mathrm{b}}$ Organic Semiconductor Centre, EaStCHEM School of Chemistry, University of St Andrews, St \\ Andrews, Fife, KY16 9ST, UK. E-mail: eli.zysman-colman@st-andrews.ac.uk ; Web: \\ http://www.zysman-colman.com; Fax:+44 (0)1334 463808; Tel:+44 (0)1334 463826.
}

${ }^{c}$ EaStCHEM School of Chemistry, University of Edinburgh

King's Buildings, Edinburgh EH9 3FJ, UK E-mail: neil.robertson@ed.ac.uk
${ }^{\mathrm{d}}$ Laboratory for Chemistry of Novel Materials, University of Mons, Place du Parc 20, B-7000 Mons, Belgium.

e Unité de Chimie Physique Théorique et Structurale \& Laboratoire de Physique du Solide, Namur Institute of Structured Matter (NISM), Université de Namur, Rue de Bruxelles, 61, 5000 Namur, Belgium.

f Unité de Chimie Physique Théorique et Structurale, Namur Institute of Structured Matter (NISM), Université de Namur, Rue de Bruxelles, 61, 5000 Namur, Belgium.

${ }^{\ddagger}$ contributed equally

\section{Abstract}

Herein, we report the use of spiro-configured fluorene-xanthene scaffolds as a novel, promising and effective strategy in thermally activated delayed fluorescence (TADF) emitter design to attain high photoluminescence quantum yields $\left(\Phi_{\mathrm{PL}}\right)$, short delayed luminescence lifetime, high external quantum 
efficiency (EQE) and minimum efficiency roll-off characteristics in organic light-emitting diodes (OLEDs). The optoelectronic and electroluminescence properties of SFX-, spiro-(fluorene-9,9'xanthene), based emitters (SFX-PO-DPA, SFX-PO-DPA-Me and SFX-PO-DPA-OMe) were investigated both theoretically and experimentally. All three emitters exhibited sky blue to green emission enabled by a Herzberg-Teller mechanism in the excited state. They possess short excited state delayed lifetimes $(<10 \mu \mathrm{s})$, high photoluminescence quantum yields $\left(\Phi_{\mathrm{PL}} \sim 70 \%\right)$ and small singlettriplet splitting energies $\left(\Delta \mathrm{E}_{\mathrm{ST}}<0.10 \mathrm{eV}\right)$ in the doped films in an mCP host matrix. The OLEDs showed some of the highest EQEs using spiro-containing emitters where maximum external quantum efficiencies (EQE $\left.\mathrm{E}_{\max }\right)$ of $11 \%$ and $16 \%$ were obtained for devices using SFX-PO-DPA and SFX-PODPA-OMe, respectively. Further, a record $\mathrm{EQE}_{\max }$ of $23 \%$ for a spiro-based emitter coupled with a small efficiency roll off $\left(19 \%\right.$ at $\left.100 \mathrm{~cd} \mathrm{~m}^{-2}\right)$ was attained with SFX-PO-DPA-Me.

\section{Introduction.}

Thermally activated delayed fluorescence (TADF) has emerged as a very popular mechanism for harvesting excitons in organic light-emitting diodes (OLEDs). ${ }^{1-3}$ Metal-free TADF materials, like state-of-the-art phosphorescent materials, can harvest $100 \%$ of the generated excitons and convert them into light. Electroluminescent devices using TADF emitters exhibit comparable efficiencies to state-ofthe-art phosphorescent OLEDs. An efficient TADF mechanism is observed from charge transfer (CT) systems that possess a small singlet-triplet energy gap $\left(\Delta \mathrm{E}_{\mathrm{ST}}\right)$ as a consequence of the small exchange integral. In donor-acceptor TADF emitter architectures, hole and electron densities in the lowest singlet and triplet excited states are spatially separated. This is frequently due to a large torsion between donor and acceptor fragments. A small $\Delta \mathrm{E}_{\mathrm{ST}}$ allows for efficient reverse intersystem crossing (RISC), resulting in an observed TADF upon photo- or electrical excitation. However, unlike phosphorescent OLEDs, TADF OLEDs frequently suffer from high efficiency roll-off at high current densities due to the relatively longer delayed exciton lifetimes $\left(\tau_{\mathrm{d}}\right)$ of the emitters. ${ }^{4}$ Many efforts have been devoted to combat this limitation by incorporating a properly designed TADF emitter. ${ }^{5-7}$ For example, it has already been established both theoretically and experimentally that a highly twisted donor-acceptor 
structure with a correspondingly small $\Delta \mathrm{E}_{\mathrm{ST}}$ is desired to achieve high RISC rates. ${ }^{8-9}$ However, this approach leads to low oscillator strength, and the resulting low radiative decay rate of the emissive $\mathrm{S}_{1}$ state usually lead to low photoluminescence quantum yields, $\Phi_{\mathrm{PL}}$. There is, therefore, a challenge in emitter design to conceive of a system that possesses both small $\Delta \mathrm{E}_{\mathrm{ST}}$ and high $\Phi_{\mathrm{PL}}$ in order to realize efficient TADF OLEDs.

Very small $\Delta \mathrm{E}_{\mathrm{ST}}$ values can be easily obtained in spiro-type compounds where donor and acceptor moieties are disposed orthogonally with respect to each other. Owing to their threedimensional structure, excimer emission is suppressed in the solid state. Spiro compounds also possess excellent thermal stability, high glass transition temperatures, and have been widely studied as optoelectronic materials. ${ }^{10-15}$ Despite having such favourable structural, thermal and optoelectronic properties that would make them highly suited as TADF emitters, there have been only a handful of reports of emitter design based on spiro architectures. Figure 1 shows all reported spiro-based TADF emitters. The first example of a spiro-based TADF compound, spiro-CN, ${ }^{16}$ is a yellow-emitting material $\left(\lambda_{\mathrm{PL}}=545 \mathrm{~nm}\right)$ with a $\Delta \mathrm{E}_{\mathrm{ST}}$ and $\tau_{\mathrm{d}}$ of $0.056 \mathrm{eV}$ and $14 \mu \mathrm{s}$, respectively, in $6 \mathrm{wt} \% \mathrm{mCP}$ doped films. The $\Phi_{\mathrm{PL}}$, however, remained only $27 \%$ and the resulting OLEDs displayed a maximum external quantum efficiency, $\mathrm{EQE}_{\max }$, of only $4.4 \%$ with $\lambda_{\mathrm{EL}}$ of $550 \mathrm{~nm}$. Upon incorporation of the donor fragment within the spiro framework as in ACRFLCN, ${ }^{17}$ the photophysical properties and the device performances were improved significantly. ACRFLCN exhibited sky blue emission $\left(\lambda_{\mathrm{PL}}=485 \mathrm{~nm}\right)$ with a vanishingly small $\Delta \mathrm{E}_{\mathrm{ST}}$ of $0.01 \mathrm{eV}$ but with a rather long $\tau_{\mathrm{d}}$ of $3.9 \mathrm{~ms}$ in $6 \mathrm{wt} \%$ doped TPSI-F films. The $\Phi_{\mathrm{PL}}$ in the solid state was nearly $67 \%$ and the OLEDs possessed an improved $\mathrm{EQE}_{\max }$ of $10.1 \%$ with $\lambda_{\mathrm{EL}}=500 \mathrm{~nm}$; however, the devices showed rather high efficiency roll-off. When both the donor and acceptor units are intrinsically a part the spiro framework as in ACRSA, ${ }^{18}$ sky blue emission was obtained $\left(\lambda_{\mathrm{PL}}=490 \mathrm{~nm}\right)$. In the $20 \mathrm{wt} \%$ DPEPO doped films, a small $\Delta \mathrm{E}_{\mathrm{ST}}$ of $0.030 \mathrm{eV}$, short $\tau_{\mathrm{d}}$ of $5.3 \mu$ s and high $\Phi_{\mathrm{PL}}$ of $81 \%$ were obtained. The resulting OLEDs showed a much-improved $\mathrm{EQE}_{\max }$ of $16.5 \%$ with a $\lambda_{\mathrm{EL}}$ of $490 \mathrm{~nm}$. However, this high $\mathrm{EQE}_{\max }$ was obtained at a very low current density of $0.01 \mathrm{~mA} \mathrm{~cm}^{-2}$. The emitter DPAA-AF,$^{19}$ where the acridine core donor unit is substituted with 
peripheral electron-donating diphenylamines, exhibited blue-green emission $\left(\lambda_{\mathrm{PL}}=490 \mathrm{~nm}\right)$, a $\Delta \mathrm{E}_{\mathrm{ST}}$ of $0.021 \mathrm{eV}, \tau_{\mathrm{d}}$ of $4.3 \mu$ s and $\Phi_{\mathrm{PL}}$ of $70 \%$ in $6 \mathrm{wt} \% \mathrm{mCP}$ doped films. The OLEDs showed an EQE $\mathrm{max}_{\max }$ of $9.6 \%$ and an $\lambda_{\mathrm{EL}}$ of $499 \mathrm{~nm}$. Two emitters OSTFCN and OSTFB were reported ${ }^{20}$ based on a modified design of the emitter ACRSA. OSTFCN and OSTFB both showed green-yellow emission with $\lambda_{\mathrm{PL}}$ of 530 and $550 \mathrm{~nm}$, accompanied by relatively larger $\Delta \mathrm{E}_{\mathrm{ST}}$ of 90 and $0.110 \mathrm{eV}$, longer $\tau_{\mathrm{d}}$ of 150 and 180 $\mu$ s and $\Phi_{\mathrm{PL}}$ of $80 \%$ and $60 \%$, respectively, in $10 \mathrm{wt} \% \mathrm{mCP}$ doped films. The OLEDs displayed high $\mathrm{EQE}_{\max }$ values of $20.4 \%$ and $18.8 \%$ with $\lambda_{\mathrm{EL}}$ of 530 and $550 \mathrm{~nm}$ for OSTFCN and OSTFB, respectively. The spiro-blocking strategy has recently been demonstrated to realize deep blue TADF emission in non-

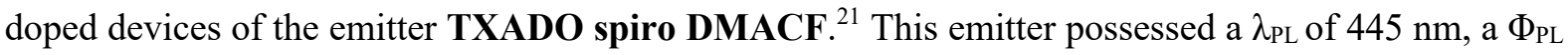
of $54 \%$, a much larger $\Delta \mathrm{E}_{\mathrm{ST}}$ of $0.280 \mathrm{eV}$ resulting in a longer $\tau_{\mathrm{d}}$ of $160 \mu \mathrm{s}$ in neat films. Non-doped OLEDs based on TXADO spiro DMACF showed deep blue emission $\left[\lambda_{\mathrm{EL}}=444 \mathrm{~nm}\right.$; CIE of $(0.16$, 0.09)]. However, the $\mathrm{EQE}_{\max }$ of the OLED was limited to $5.3 \%$ and device suffered from severe efficiency roll-off at high current densities. Recently, emitter SAF-3CN, a slightly modified design of ACRFLCN was reported, ${ }^{22}$ where an additional electron-withdrawing cyano group was introduced at the $\mathrm{C} 4$ site of the fluorene moiety (Figure 1). SAF-3CN showed green emission with $\lambda_{\mathrm{PL}}$ of $540 \mathrm{~nm}$, $\Phi_{\mathrm{PL}}$ of $65 \%$, a small $\Delta \mathrm{E}_{\mathrm{ST}}$ of $0.01 \mathrm{eV}$ and $\tau_{\mathrm{d}}$ of $21.5 \mu \mathrm{s}$ in $2 \mathrm{wt} \%$ doped films in CBP host. The OLEDs exhibited green emission $\left[\lambda_{\mathrm{EL}}=532 \mathrm{~nm}\right.$; $\mathrm{CIE}$ of $\left.(0.36,0.57)\right]$ and an $\mathrm{EQE}_{\max }$ of $19.4 \%$, which reduced to $14.4 \%$ at $1000 \mathrm{~cd} \mathrm{~m}^{-2}$. The ACRFLCN moiety was modified in emitter QAFCN to generate a more rigid donor structure in order to enhance both the $\Phi_{\mathrm{PL}}$ and RISC in the solid state. ${ }^{23}$ QAFCN showed sky-blue emission with $\lambda_{\mathrm{PL}}=490 \mathrm{~nm}, \Phi_{\mathrm{PL}}$ of $71 \%$, and $\Delta \mathrm{E}_{\mathrm{ST}}$ of $0.19 \mathrm{eV}$ in $12 \mathrm{wt} \%$ doped films in DPEPO host. The resulting sky-blue OLEDs $\left[\lambda_{\mathrm{EL}}=488 \mathrm{~nm}\right.$; CIE of $\left.(0.19,0.35)\right]$ showed an $\mathrm{EQE}_{\max }$ of $17.9 \%$, which reduced significantly to $9.5 \%$ at high current density of $100 \mathrm{~cd} \mathrm{~m}^{-2}$. From this analysis, it is evident that spiro-configured architectures incorporated into the emitter design beneficially contribute towards small $\Delta \mathrm{E}_{\mathrm{ST}}$ values; however, to concurrently maintain a high $\Phi_{\mathrm{PL}}$ and a short $\tau_{\mathrm{d}}$ for this class of emitter remains a major challenge. 


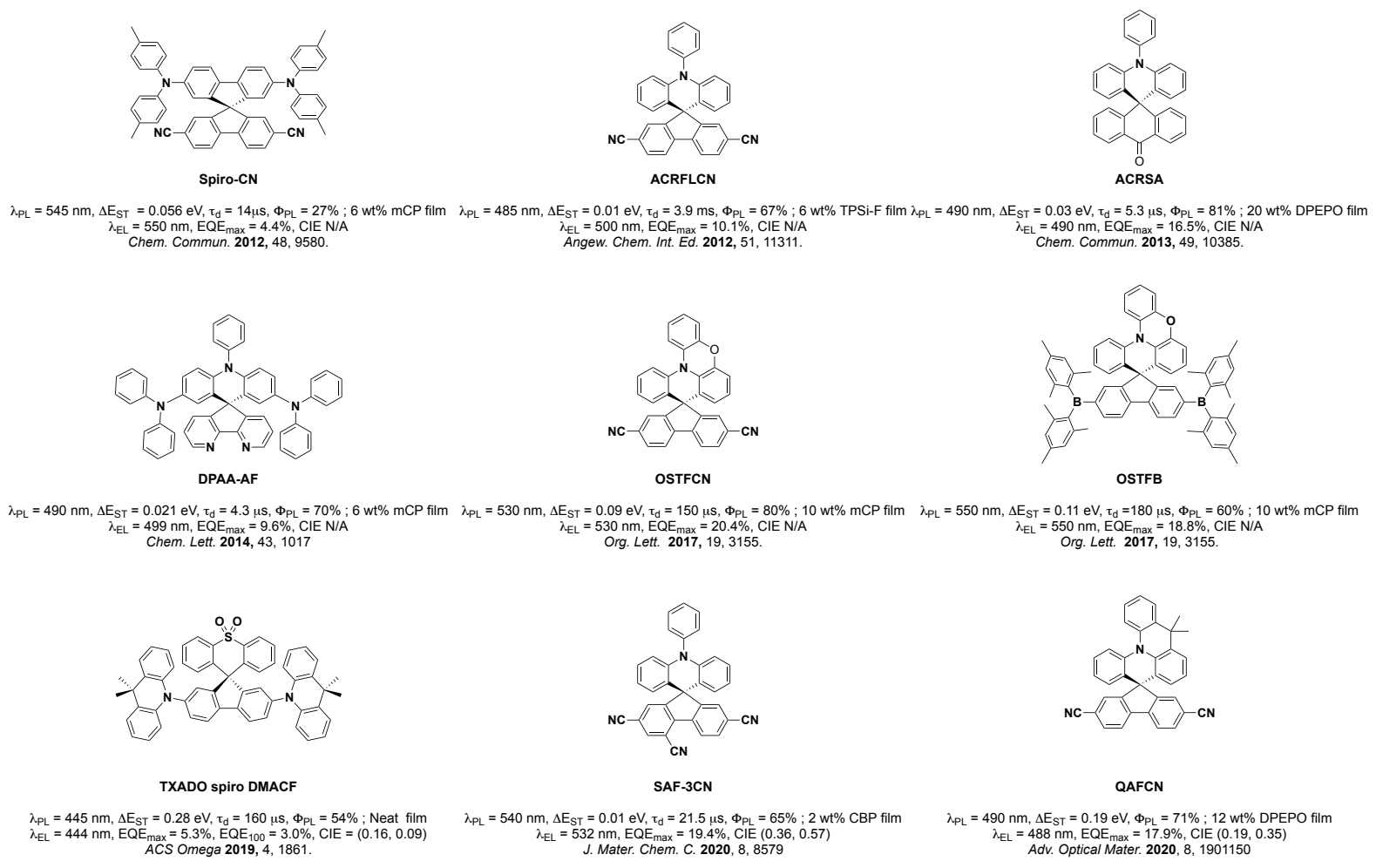

Figure 1. Chemical Structures and performances of literature spiro-based TADF compounds.

From the synthetic point of view all the previously reported structures are very similar since they have either non-aromatic acceptor as with nitriles (Spiro-CN, ACRFLCN, OSTFCN, SAF-3CN and QAFCN) or the acceptor substituent is incorporated into one of the spiro $\pi$-systems (ACRSA, OSTFB, DPAA-AF and TXADO spiro DMACF). This may simplify, in some cases, the synthesis of these materials but it also restricts molecular design to a small number of donor-acceptor pairs, thereby limiting the utilization of the great potential associated with the spiro architecture for designing highly efficient TADF emitters.

We report here a promising molecular design strategy to concurrently obtain a small $\Delta \mathrm{E}_{\mathrm{ST}}$, high $\Phi_{\mathrm{PL}}$, short $\tau_{\mathrm{d}}$ in TADF emitters that should translate to OLEDs with high EQEs and improved efficiency roll-off. We developed three highly efficient TADF emitters by incorporating an SFX, spiro-(fluorene9,9'-xanthene), system ${ }^{24}$ where a fluorene unit decorated with weakly electron-accepting phosphine 
oxides is spiro-linked to a xanthene core containing pendant diarylamine donors (Figure 2). As with most of the spiro compounds, our SFX-based emitters exhibit very weak spatial overlap between the frontier molecular orbitals due to the mutually orthogonal donor (D) and acceptor (A) orientation, so that the lowest electronic excitation should be 'dark'. However, vibronic coupling in the lowest excited state can result in intensity borrowing from higher-lying bright states, imparting the emissive state with non-negligible oscillator strength and radiative decay rates.

The presented materials and synthetic approach circumvent current spiro-based molecular design limitations by exploiting electronic separation of unsubstituted fluorene and xanthene $\pi$-systems thus facilitating easy, stepwise introduction of aromatic acceptors on one half, followed by bromination of the other half that then permits facile introduction of the aromatic donors. We tuned the degree of overlap between HOMO and LUMO moieties through the choice of donor system in order to achieve efficient radiative decay and short $\tau_{\mathrm{d}}$ while maintaining a small $\Delta \mathrm{E}_{\mathrm{ST}}$. Further, the inherent rigidity of a spiro centre also restricts both intramolecular non-radiative decay to the ground state from either the first singlet $\left(\mathrm{S}_{1}\right)$ or triplet $\left(\mathrm{T}_{1}\right)$ excited states and intermolecular interactions in the solid state, which limits bimolecular recombination processes such as triplet-triplet and triplet-polaron annihilations, contributing to the high $\Phi_{\mathrm{PL}}$. Three novel emitters, SFX-PO-DPA, SFX-PO-DPA-Me and SFX-PODPA-OMe were synthesized, and their optoelectronic properties were investigated and corroborated by a thorough computational study. All three TADF emitters showed short $\tau_{\mathrm{d}}(<10 \mu \mathrm{s})$, high $\Phi_{\mathrm{PL}}(\sim 70 \%)$ and modest $\Delta \mathrm{E}_{\text {ST }}$ (ca. $0.10 \mathrm{eV}$ ) in doped films in a mCP host matrix. The OLEDs incorporating these emitters exhibited sky-blue to green emission. $\mathrm{EQE}_{\max }$ values approaching $11 \%$ and $16 \%$ were obtained for SFX-PO-DPA and SFX-PO-DPA-OMe, respectively. Impressively, the OLED with SFX-PODPA-Me as the emitter showed an $\mathrm{EQE}_{\max }$ of $23 \%$ combined with low efficiency roll-off $\left(\mathrm{EQE}_{100}=\right.$ $19 \%$ at $100 \mathrm{~cd} \mathrm{~m}^{-2}$ ). This device performance validates the SFX spiro design and places it as the most efficient OLED among those employing spiro-configured TADF emitters to date. 


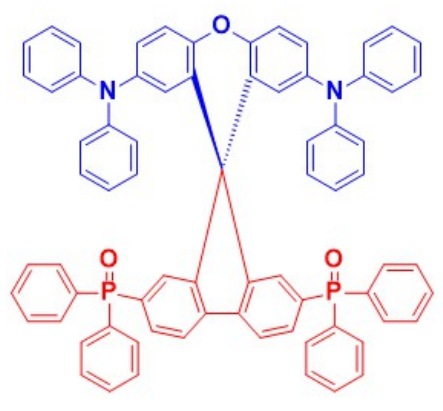

SFX-PO-DPA

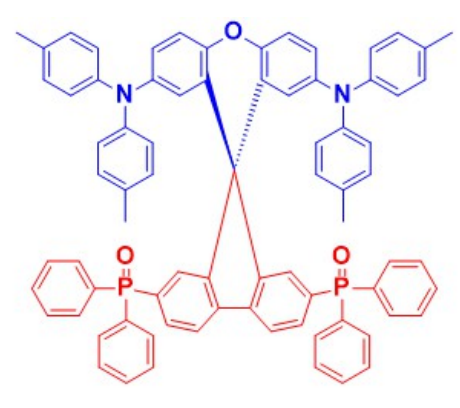

SFX-PO-DPA-Me

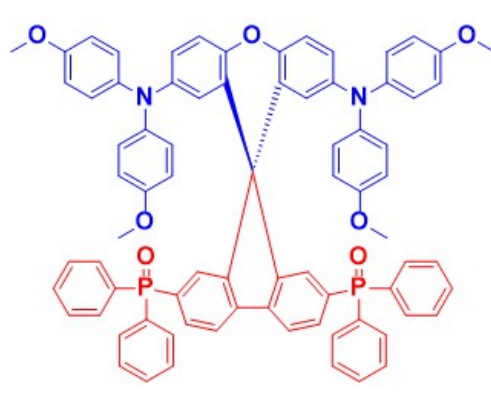

SFX-PO-DPA-OMe

Figure 2. Chemical Structures of SFX-PO-DPA, SFX-PO-DPA-Me and SFX-PO-DPA-OMe.

\section{Results and Discussion}

\section{Synthesis}

The synthetic procedures for the preparation of SFX-based emitters are presented in Scheme $\mathbf{1 .}$ The starting material, 2,7-dibromospiro[fluorene-9,9'-xanthene] SFX-Br was prepared according to a modified protocol $^{25}$ reported for the large-scale synthesis of spiro[fluorene-9,9'-xanthene]. ${ }^{26}$ Spiro[fluorene-9,9'-xanthene]-2,7-diylbis(diphenylphosphine oxide) SFX-PO was synthesized according to the literature. ${ }^{27}$ The selective electrophilic aromatic dibromination of the xanthene ring of the SFX-PO scaffold was achieved by utilization of $N$-bromosuccinimide (NBS) in glacial acetic acid at elevated temperature $\left(110^{\circ} \mathrm{C}\right)$ in a good yield of $72 \%$; room temperature reaction in glacial acetic acid and acetonitrile gave only starting material. The successful conditions left the deactivated fluorene and diphenylphosphine oxide rings untouched by taking advantage of electronic separation from the more reactive xanthene half, even in the presence of excess (4 equiv.) NBS. The total yield after three steps for this SFX-PO-Br intermediate was 53\%. The emitters were obtained by Buchwald-Hartwig coupling of SFX-PO-Br and three different diphenylamines: diphenylamine, 4,4'dimethyldiphenylamine and 4,4'-dimethoxydiphenylamine. Three different combinations of catalyst, base and ligand were evaluated: $\mathrm{Pd}_{2}(\mathrm{dba})_{3}, \mathrm{P}(t-\mathrm{Bu})_{3}$ and $\mathrm{NaO} t$ - $\mathrm{Bu}$ in toluene at $110{ }^{\circ} \mathrm{C}$ for $16 \mathrm{~h}$ gave a mixture of products. $\mathrm{Pd}(\mathrm{OAc})_{2},\left[(t-\mathrm{Bu})_{3} \mathrm{PH}\right] \mathrm{BF}_{4}$ and $\mathrm{NaO} t-\mathrm{Bu}$ in toluene at $110{ }^{\circ} \mathrm{C}$ for $16 \mathrm{~h}$ gave only starting materials. However, utilization of cesium carbonate as a base with $\mathrm{Pd}_{2}(\mathrm{dba})_{3}$ as the catalyst and $\mathrm{P}(t-\mathrm{Bu})_{3}$ as the ligand successfully yielded the desired products with moderate yields of $45 \%, 53 \%, 44 \%$ for SFX-PO-DPA, SFX-PO-DPA-Me and SFX-PO-DPA-OMe, respectively. As has been previously 
reported, the use of a weaker base like cesium carbonate provides better compatibility with basesensitive functional groups. ${ }^{28}$

This approach permits tuning of the optoelectronic properties of the emitters by appropriate choice of donor-acceptor combinations. Here, we tested only diphenylphosphine oxide as an acceptor and focused on the influence on the different diphenylamine substituents. However, the results indicate great influence of the type of the substituent, and hence a wide opportunity for fine-tuning and perspective for further development.
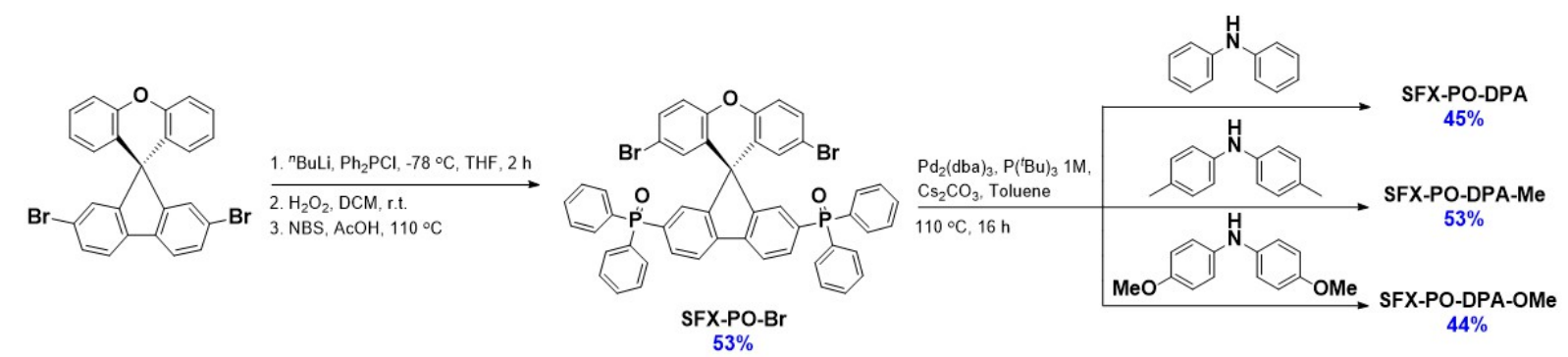

Scheme 1. Synthetic routes for SFX-PO-Br-starting material and three studied emitters: SFX-PODPA, SFX-PO-DPA-Me and SFX-PO-DPA-OMe.

\section{Crystal Structures}

In order to gain further insights into these compounds, the structures of SFX-PO-DPA and SFX-PO-DPA-Me have been studied by single-crystal X-ray diffraction (Figure S1). The data obtained from X-ray diffraction analysis are summarized in Table S1. SFX-PO-DPA formed light-yellow rodshaped crystals and was found to crystallize in the monoclinic space group $C 2 / c$ when recrystallised from a mixture of methanol and dichloromethane by solvent layering. SFX-PO-DPA-Me formed paleyellow block-shaped crystals in the monoclinic I2/a space group when recrystallised from a mixture of hexane and dichloromethane by evaporation. Recrystallization of SFX-PO-DPA-Me from the same mixture as for SFX-PO-DPA (i.e., methanol and dichloromethane by solvent layering) led to formation of pale-yellow block-shaped crystals in the same spaced group $C 2 / c$. The crystal structures and interactions between the materials and co-crystallized solvent molecules are shown in Figure 3. As depicted in Figure 3, co-crystallized solvent molecules have an influence on the dihedral angle between 
fluorene and xanthene in the spiro-structure. When dichloromethane and hexane are present in the SFXPO-DPA-Me molecule, the dihedral angle is $86.7^{\circ}$. However, switching to a more polar solvent system (dichloromethane and methanol), the angle increases to $89.5^{\circ}$. With the same solvent system (dichloromethane and methanol) applied to the crystallization of SFX-PO-DPA only two methanol molecules were incorporated in the structure leading to a dihedral angle of $87.6^{\circ}$. The variation in the value of the dihedral angle as a function of the environment will have an influence on through bond interactions between the orthogonally disposed donor and acceptor units, thereby affecting the photophysical properties of these spiro-based structures.

a)

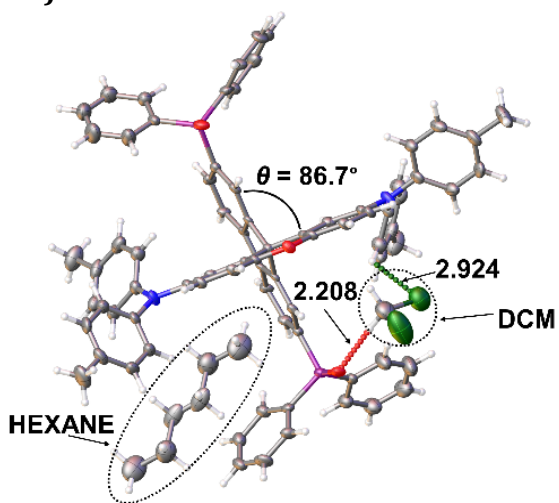

b)

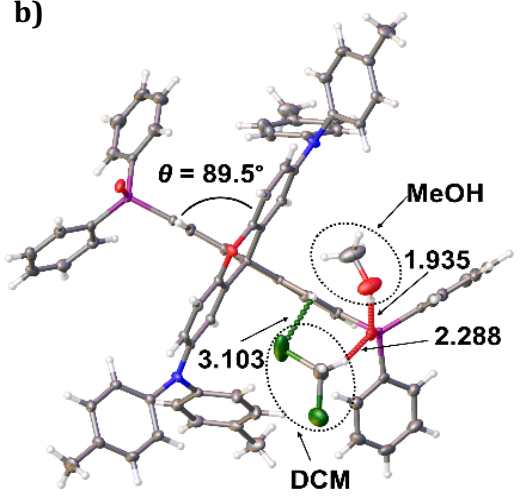

c)

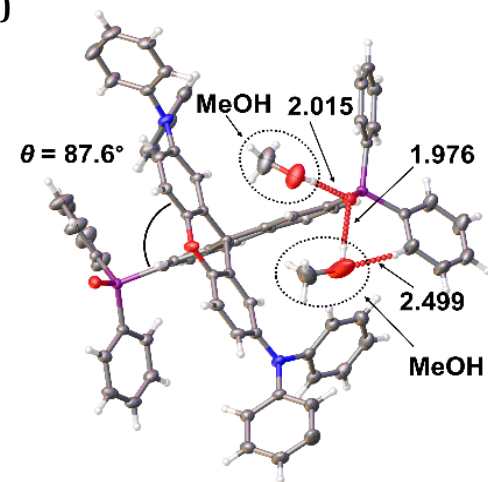

Figure 3. Crystal structures and dihedral angles of (a) SFX-PO-DPA-Me recrystallized from

DCM/Hexane, (b) SFX-PO-DPA-Me recrystallized from DCM/MeOH and (c) SFX-PO-DPA recrystallized from DCM:Hexane. Heteroatoms: $\mathrm{O}$, red; N, blue; P, violet; $\mathrm{Cl}$, green.

\section{Electrochemical Properties.}

The HOMO and LUMO energy levels of the three emitters were inferred from an analysis of the cyclic voltammetry (CV) and differential pulse voltammetry (DPV) measurements obtained in dichloromethane (for oxidation) and N,N-dimethylformamide (for reduction). The CVs and DPVs are shown in Figure $\mathbf{4}$ and the data are summarized in Table 1. Each of the emitters exhibits two highly reversible oxidation waves, reflecting sequential oxidation of each of the two donor moieties. As predicted by the theoretical calculations (Figure 9, vide infra), the HOMO in all three compounds is 
delocalized across the two donor moieties through the xanthene bridge. Such delocalization can significantly stabilize the radical cation formed upon the first oxidation. As a result, removal of the second electron becomes less difficult than for a less-delocalized HOMO due in part to lower Coulombic repulsion and this is manifested in the fairly close spacing of the two CV oxidation waves. ${ }^{29}$ For SFX-PO-DPA, $E_{o x}$, the peak oxidation potential as determined from DPV, is $0.76 \mathrm{~V}$. The oxidation potentials shifted cathodically with increasing donor strength to $0.73 \mathrm{~V}$ and $0.60 \mathrm{~V}$ for SFX-PO-DPAMe and SFX-PO-DPA-OMe, respectively, consistent with the picture obtained by DFT calculations (Figure 9, vide infra). These values are almost identical to the oxidation potentials of related tetrasubstituted hole transport materials, such as SFX-MeOTAD (0.52 V vs SCE) with four dimethoxydiphenylamine groups and SFX-TAD (0.68 V vs SCE) with four diphenylamine groups, reported by us previously. ${ }^{26}$ This indicates no influence on the oxidation potential from the diphenylphosphine oxide-modified fluorene fragment. The HOMO energies were determined to be $5.56 \mathrm{eV},-5.53 \mathrm{eV}$ and $-5.40 \mathrm{eV}$ for SFX-PO-DPA, SFX-PO-DPA-Me and SFX-PO-DPA-OMe, respectively. The decrease in HOMO energy is associated with the increase in mesomeric effect with increasing electron-donating strength of the amine donor groups. Only one irreversible reduction wave was observed for all three compounds. LUMO energies were inferred from the reduction potentials obtained by DPV measurements in DMF and were found to be $-2.94 \mathrm{eV},-3.03 \mathrm{eV}$ and $-3.15 \mathrm{eV}$ for SFX-PO-DPA, SFX-PO-DPA-Me and SFX-PO-DPA-OMe, respectively. Unexpectedly, we observed a small stabilization of the LUMO with increasing donor strength associated with an increase in stabilizing inductive effect by substituting DPA successively with Me and OMe substituents, whilst the mesomeric effect is negligible due to the complete localization of the LUMO on the acceptor (see molecular modelling section). 


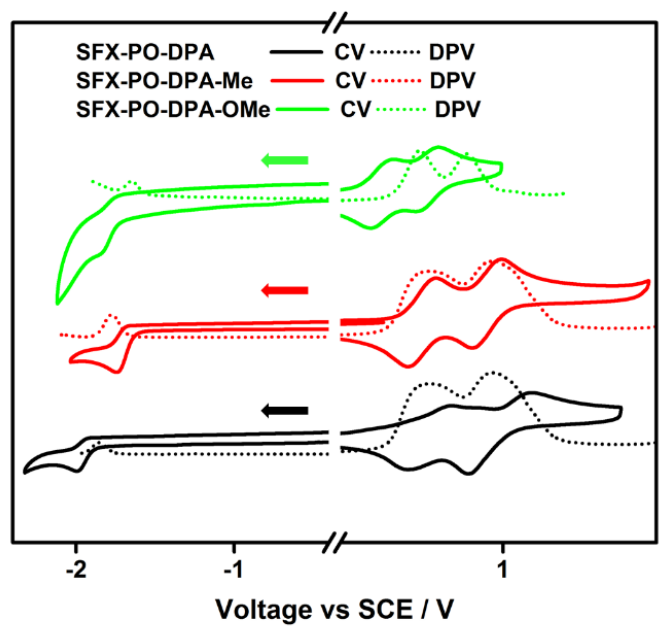

Figure 4. Cyclic Voltammograms and Differential Pulse Voltammograms of SFX-PO-DPA, SFX-PODPA-Me and SFX-PO-DPA-OMe (Oxidation and reduction was performed in degassed DCM and DMF, respectively; scan rate: $100 \mathrm{mV} \mathrm{s}^{-1}$ ).

Table 1. Electrochemical properties of SFX-PO-DPA, SFX-PO-DPA-Me and SFX-PO-DPA-OMe.

\begin{tabular}{|c|c|c|c|c|c|}
\hline & $E_{\text {ox }}$ a $/ \mathbf{V}$ & $E_{\text {red }} \mathbf{b} / \mathbf{V}$ & HOMO $^{\text {a,c }} / \mathbf{e V}$ & LUMO $^{\text {b,c }} / \mathbf{e V}$ & $\Delta \mathbf{E}_{\text {redox }}{ }^{\mathrm{d}} / \mathrm{eV}$ \\
\hline SFX-PO-DPA & 0.76 & -1.86 & -5.56 & -2.94 & 2.62 \\
\hline SFX-PO-DPA-Me & 0.73 & -1.77 & -5.53 & -3.03 & 2.50 \\
\hline SFX-PO-DPA-OMe & 0.60 & -1.65 & -5.40 & -3.15 & 2.25 \\
\hline \multicolumn{6}{|c|}{ 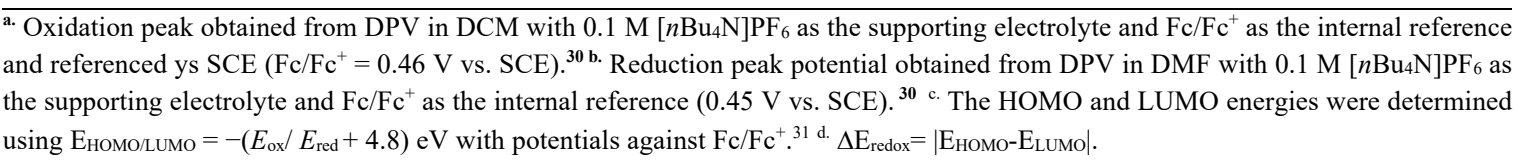 } \\
\hline
\end{tabular}

\section{Photophysical Properties.}

Figure 5a shows the UV-Vis absorption and photoluminescence (PL) spectra of the three emitters in toluene (PhMe) and the data are summarized in Table 2 . All three compounds exhibit similar optical absorption and possess a series of highly absorbing bands from $290 \mathrm{~nm}$ to $320 \mathrm{~nm}$, which we attribute from Time-Dependent Density Functional Theory (TD-DFT) calculations to high-oscillatorstrength locally excited electronic transitions to $S_{n}(n>1)$ states, while, as expected from its strong 
charge-transfer character, the oscillator strength for the promotion to $S_{1}$ is much smaller (Tables $\mathbf{S} \mathbf{4}$ and 5, Figure S14). The PL spectra in degassed PhMe are broad and unstructured, typical of emission from a CT state. A red-shift in the emission from SFX-PO-DPA $\left(\lambda_{\mathrm{PL}}=490 \mathrm{~nm}\right)$ to SFX-PO-DPA-Me $\left(\lambda_{\mathrm{PL}}\right.$ $=492 \mathrm{~nm})$ and to SFX-PO-DPA-OMe $\left(\lambda_{\mathrm{PL}}=514 \mathrm{~nm}\right)$ is consistent with the decrease in the HOMOLUMO gap, an observation corroborated by theoretical calculations (see Figure 9, vide infra). The $\Phi_{\mathrm{PL}}$ values of $56 \%, 80 \%$ and $68 \%$ for SFX-PO-DPA, SFX-PO-DPA-Me and SFX-PO-DPA-OMe, respectively, in degassed PhMe varied significantly as a function of the pendant groups on the donor amine. Emission was dramatically quenched, with much reduced $\Phi_{\mathrm{PL}}$ of $19 \%, 38 \%$ and $25 \%$ SFX-PODPA, SFX-PO-DPA-Me and SFX-PO-DPA-OMe, respectively, upon exposure to air, an indication of triplet harvesting in the absence of oxygen.

(a)

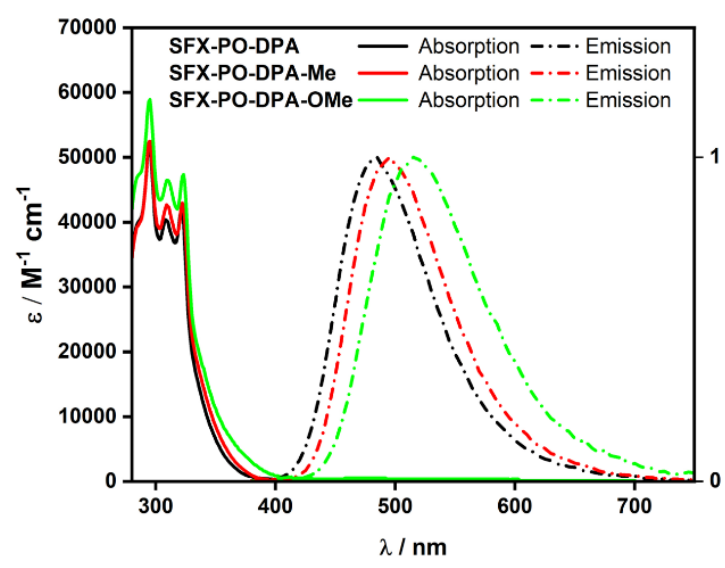

(b)

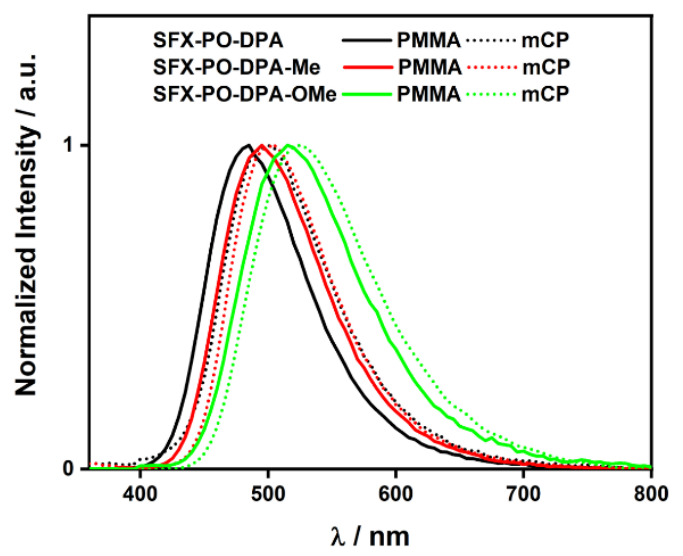

Figure 5. (a) UV-Vis absorption and PL spectra in PhMe and (b) PL spectra in PMMA and mCP thin films of SFX-PO-DPA (10 wt $\%$ in both), SFX-PO-DPA-Me (10 wt $\%$ in PMMA and $15 \mathrm{wt} \%$ in mCP) and SFX-PO-DPA-OMe (10 wt $\%$ in both). $\lambda_{\text {exc }}=360 \mathrm{~nm}$.

We next investigated the PL behavior of the three compounds in the solid state as doped films in both PMMA and mCP (Figure 5b). Irrespective of the host, the emission profiles remained broad and unstructured. In solution-processed $10 \mathrm{wt} \%$ doped films in PMMA, all three compounds exhibited bluegreen to green emission with $\lambda_{\text {PL }}$ of $490 \mathrm{~nm}$ for SFX-PO-DPA, $495 \mathrm{~nm}$ for SFX-PO-DPA-Me and 515 $\mathrm{nm}$ for SFX-PO-DPA-OMe, results in line with those observed in toluene. However, the $\Phi_{\mathrm{PL}}$ suffered 
in the PMMA films and values of $10 \%, 38 \%$ and $25 \%$ were obtained for SFX-PO-DPA, SFX-PODPA-Me and SFX-PO-DPA-OMe, respectively. Time-resolved PL measurements in PMMA revealed bi-exponential decay profiles for all three emitters. SFX-PO-DPA, SFX-PO-DPA-Me and SFX-PODPA-OMe possessed prompt lifetimes, $\tau_{\mathrm{p}}$, of $92 \mathrm{~ns}, 126 \mathrm{~ns}$ and $156 \mathrm{~ns}$, respectively, and delayed lifetimes, $\tau_{\mathrm{d}}$, of $10 \mu \mathrm{s}, 9.4 \mu \mathrm{s}$ and $6.4 \mu \mathrm{s}$, respectively. Based on the emission energies of the three emitters, $\mathrm{mCP}$ was chosen as the host matrix for OLEDs. Optimum doping concentrations of $10 \mathrm{wt} \%$ for SFX-PO-DPA and SFX-PO-DPA-OMe, and $15 \mathrm{wt} \%$ for SFX-PO-DPA-Me were determined based on a concentration-dependence study of absolute $\Phi_{\mathrm{PL}}$ values of vacuum-deposited films (Table S2, ESI). At these concentrations, slightly red-shifted emission maxima of $500 \mathrm{~nm}$ for SFX-PO-DPA and SFX-PO-DPA-Me, and $520 \mathrm{~nm}$ for SFX-PO-DPA-OMe were observed along with much enhanced $\Phi_{\mathrm{PL}}$ values of $50 \%, 70 \%$ and $58 \%$, respectively, compared to the measurements in PMMA. Time-resolved PL measurements again showed bi-exponential decay behavior for all three emitters, with an enhanced contribution from the delayed emission (Figure 7). In comparison to the non-polar host PMMA, faster transient PL dynamics were observed in $\mathrm{mCP}$ both in terms of prompt and delayed lifetimes. Prompt lifetimes of $81 \mathrm{~ns}(43 \%), 49 \mathrm{~ns}(18 \%)$ and $100 \mathrm{~ns}(40 \%)$, and delayed lifetimes of 8.4 $\mu$ s $(57 \%), 8.3 \mu$ s $(82 \%)$ and $6.2 \mu$ s $(60 \%)$ were obtained for SFX-PO-DPA, SFX-PO-DPA-Me and SFX-PO-DPA-OMe, respectively, showing that delayed fluorescence also occurs in the mCP host. The long radiative lifetimes measured for the prompt component of these compounds support the emission from CT states with limited overlap between hole and electron densities. 


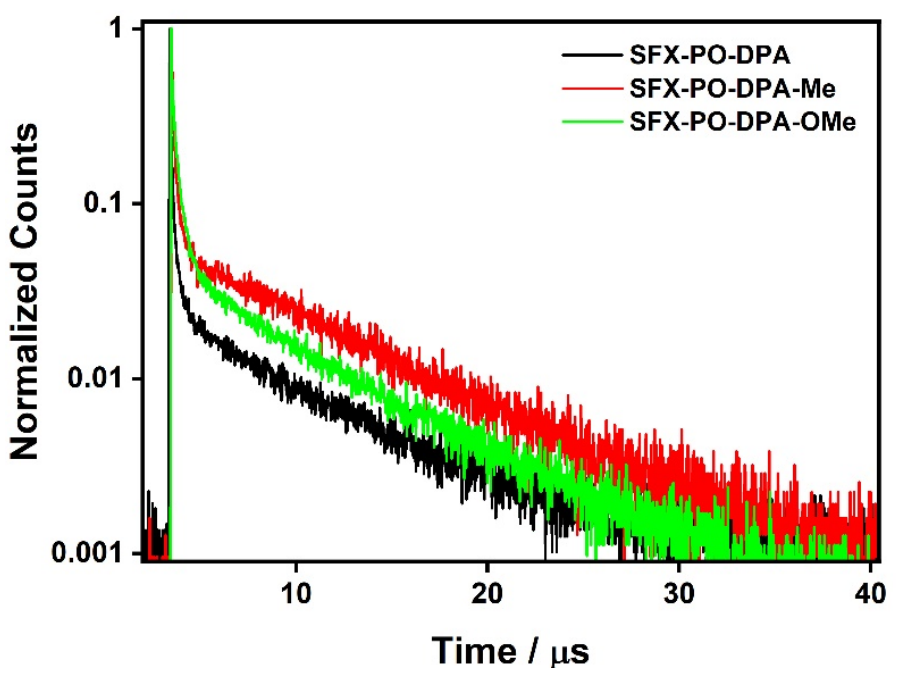

Figure 6. Normalized time-resolved PL of vacuum-deposited mCP films with $10 \mathrm{wt} \%$ SFX-PO-DPA, $15 \mathrm{wt} \%$ SFX-PO-DPA-Me and $10 \mathrm{wt} \%$ SFX-PO-DPA-OMe at room temperature. $\lambda_{\mathrm{exc}}=378 \mathrm{~nm}$.

To further confirm the TADF character of these emitters, we next studied the temperature dependence of the time-resolved emission decays of the mCP doped films containing the three emitters. An increase in the relative intensity of the delayed component of the lifetime with increasing temperature provides direct evidence to support the TADF character of these spiro-based emitters (Figure 7a-c). The $\Delta E_{\mathrm{ST}}$ values in $\mathrm{mCP}$ of the three emitters were determined from the difference in the energies of the fluorescence and phosphorescence spectra obtained from the onset of prompt and delayed emission, respectively, at $77 \mathrm{~K}$ (Figure 9a-c). Very small $\Delta \mathrm{E}_{S \mathrm{ST}}$ values of $0.05 \mathrm{eV}, 0.02 \mathrm{eV}$ and $0.01 \mathrm{eV}$ and $\mathrm{S}_{1}$ energies of $2.85 \mathrm{eV}, 2.80 \mathrm{eV}$ and $2.75 \mathrm{eV}$ were measured for SFX-PO-DPA, SFX-PODPA-Me and SFX-PO-DPA-OMe respectively, in line with theory (Table S4) and confirming their strong potential as efficient TADF emitters in the solid state. 

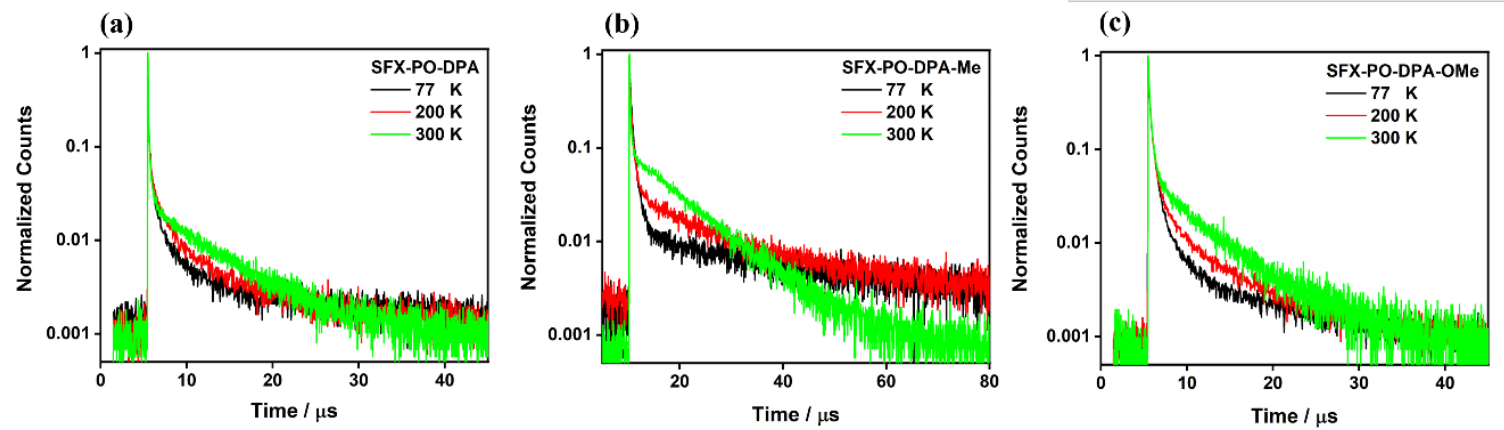

Figure 7. Temperature-dependent time-resolved PL decay traces of doped films in $\mathrm{mCP}$ with (a) 10 $\mathrm{wt} \%$ SFX-PO-DPA (b) $15 \mathrm{wt} \%$ SFX-PO-DPA-Me and (c) $10 \mathrm{wt} \%$ SFX-PO-DPA-OMe $\left(\lambda_{\mathrm{exc}}=378\right.$ $\mathrm{nm})$.

In order to understand the origin of the short $\tau_{\mathrm{d}}$ and high $\Phi_{\mathrm{PL}}$, we calculated the rate constants for the radiative decay from the singlet state $\left(k_{r}{ }^{S}\right)$, RISC $\left(k_{r I S C}\right)$ and non-radiative decay from the triplet state $\left(k_{n r}{ }^{T}\right)$. It should be noted that the rate constants for radiative and non-radiative processes can only be explicitly calculated assuming a monoexponential decay of the emission. For bi-exponential decay, we calculated the corresponding rates making a set of assumptions as described previously (see ESI, section 5.2 for details). ${ }^{32}$ All three emitters exhibited similar $k_{\mathrm{r}}{ }^{\mathrm{S}}$ on the order of $2.3-2.6 \times 10^{6} \mathrm{~s}^{-1}$ (Table S3). Importantly, $k_{\mathrm{RISC}}$, which is a crucial parameter responsible for an efficient TADF mechanism, remained higher than the competing $k_{n r}{ }^{T}$ rate constant for all three emitters, implying that the thermal upconversion of excitons from $T_{1}$ to $S_{1}$ is preferred over non-radiative decay from the $T_{1}$ state. Furthermore, the relatively higher value of $k_{\mathrm{RISC}}$ coupled with a very small $\Delta \mathrm{E}_{\mathrm{ST}}$ in SFX-PO-DPA-Me is indicative of a faster and more efficient RISC mechanism in this emitter, which results in the highest $\Phi_{\mathrm{PL}}$ values observed across all media. Such short $\tau_{\mathrm{d}}$ values and large $k_{\mathrm{RISC}}$ have been shown to play a crucial role in realizing low efficiency roll-off in the devices by reducing the probability of triplet exciton quenching mechanisms. ${ }^{33}$ 
(a)

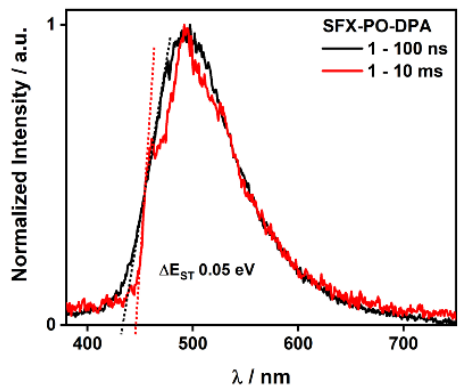

(b)

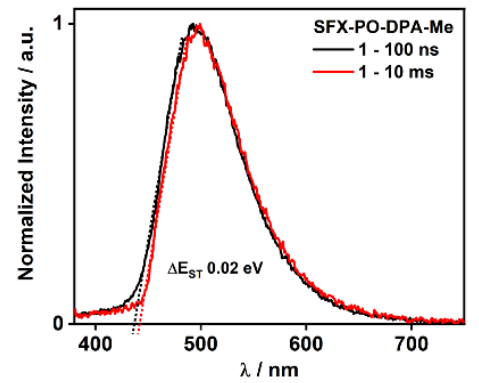

(c)

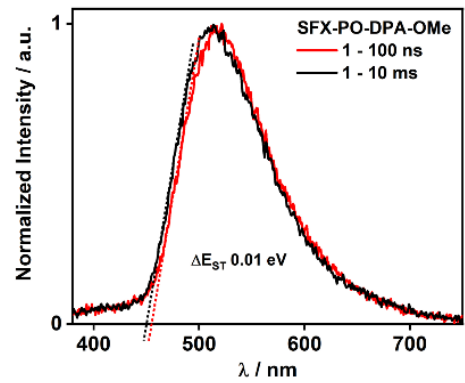

Figure 8. Prompt and delayed (by $1 \mathrm{~ms}$ ) spectra (at $77 \mathrm{~K}$ ) of doped films in $\mathrm{mCP}$ with (a) $10 \mathrm{wt} \%$

SFX-PO-DPA (b) $15 \mathrm{wt} \%$ SFX-PO-DPA-Me and (c) $10 \mathrm{wt} \%$ SFX-PO-DPA-OMe $\left(\lambda_{\mathrm{exc}}=355 \mathrm{~nm}\right)$.

Table 2. Photophysical properties of SFX-PO-DPA, SFX-PO-DPA-Me and SFX-PO-DPA-OMe.

\begin{tabular}{|c|c|c|c|c|c|c|c|c|c|c|}
\hline & $\lambda_{\mathrm{abs}}^{\mathrm{a}}$ & $\lambda_{\mathrm{PL}}{ }^{\mathrm{a}} / \lambda_{\mathrm{PL}}{ }^{\mathrm{c}}$ & $\Phi_{\mathrm{PL}}{ }^{\mathrm{b}}$ & $\Phi_{\mathrm{PL}}{ }^{\mathrm{c}}$ & $\tau_{p}^{c} /$ & $\tau_{d}^{c}$ & $S_{1} / T_{1}{ }^{d}$ & $\Delta \mathrm{E}_{\mathrm{ST}}{ }^{\mathrm{d}}$ & $k_{\mathrm{RISC}}{ }^{\mathrm{e}}$ & $k_{\mathrm{nr}}{ }^{\mathrm{T} e}$ \\
\hline & $/ \mathbf{n m}$ & $/ \mathbf{n m}$ & $1 \%$ & $/ \%$ & $/ \mathrm{ns}$ & $/ \mu \mathrm{s}$ & $/ \mathrm{eV}$ & $/ \mathrm{eV}$ & $/ 10^{5} \mathrm{~s}^{-1}$ & $/ 10^{4} \mathrm{~s}^{-1}$ \\
\hline $\begin{array}{c}\text { SFX-PO- } \\
\text { DPA }\end{array}$ & $\begin{array}{l}290, \\
300, \\
320\end{array}$ & $490 / 500$ & $\begin{array}{c}56 \\
(19)\end{array}$ & 50 & 49 & 8.4 & $\begin{array}{l}2.85 / 2 . \\
80\end{array}$ & 0.05 & 2.64 & 7.53 \\
\hline $\begin{array}{l}\text { SFX-PO- } \\
\text { DPA-Me }\end{array}$ & $\begin{array}{c}290, \\
301,320\end{array}$ & $492 / 500$ & $\begin{array}{c}80 \\
(38)\end{array}$ & 70 & 81 & 8.3 & $\begin{array}{l}2.80 / 2 \\
78\end{array}$ & 0.02 & 2.57 & 4.20 \\
\hline $\begin{array}{c}\text { SFX-PO- } \\
\text { DPA-OMe }\end{array}$ & $\begin{array}{l}290, \\
300, \\
320\end{array}$ & $514 / 520$ & $\begin{array}{c}68 \\
(25)\end{array}$ & 58 & 100 & 6.2 & $\begin{array}{l}2.75 / 2 . \\
74\end{array}$ & 0.01 & 2.32 & 8.79 \\
\hline
\end{tabular}

${ }^{\mathrm{a}}$ In PhMe at $298 \mathrm{~K} .{ }^{\mathrm{b}}$ Quinine sulfate $(0.5 \mathrm{M})$ in $\mathrm{H}_{2} \mathrm{SO}_{4}(\mathrm{aq})$ was used as the reference $\left(\Phi_{\mathrm{PL}}: 54.6 \%, \lambda_{\text {exc }}=360 \mathrm{~nm}\right) .{ }^{34}$ Values quoted are in degassed solutions, which were prepared by three freeze-pump-thaw cycles. Values in parentheses are for aerated solutions, which were prepared by bubbling air for $10 \mathrm{~min} .{ }^{\mathrm{c}}$ Thin films were prepared by vacuum depositing 10 wt.\% (15 wt.\% for SFX-PO-DPA-Me) doped samples in $\mathrm{mCP}$ and values were determined using an integrating sphere $\left(\lambda_{\text {exc }}=340 \mathrm{~nm}\right)$; degassing was done by $\mathrm{N}_{2}$ purge for 10 minutes. Determined from the onset of the prompt and delayed emission spectra of $10 \mathrm{wt} \%$ (15 wt.\% for SFX-PO-DPA-Me) doped films in mCP, measured at $77 \mathrm{~K}\left(\lambda_{\text {exc }}=355 \mathrm{~nm}\right)$. ${ }^{\mathrm{e}}$ In doped films in mCP matrix. See text for doping concentrations.

\section{Molecular Modelling}

Characterization of the electronic structure and optoelectronic properties of the three SFX spiro compounds has been carried on with the help of DFT calculations. More specifically, we used the methodology developed previously that offers a particularly accurate description of the electronic structure of materials for OLED applications (Figure 9), namely the PBE0 functional was adopted and the excited-state properties calculated within the Tamm-Dancoff approximation. ${ }^{35}$ The increasingly 
shallower HOMO level along the series SFX-PO-DPA, SFX-PO-DPA-Me and SFX-PO-DPA-OMe is consistent with the increasing donor strength of the diarylamine donor. For each emitter, the LUMO is localized exclusively on the fluorene core with no contribution from the pendant phosphine oxides and is only slightly affected by stabilizing inductive effects of the side groups attached to the DPA core. $\mathrm{S}_{1}$ energies decrease along the series from $2.77 \mathrm{eV}$ for SFX-PO-DPA to $2.34 \mathrm{eV}$ for SFX-PO-DPAOMe. Very small $\Delta \mathrm{E}_{\mathrm{ST}}$ values of $0.0038 \mathrm{eV}$ for SFX-PO-DPA and SFX-PO-DPA-Me and $0.00051 \mathrm{eV}$ for SFX-PO-DPA-OMe reflect the near orthogonal arrangement of the donor and acceptor groups. We determined the nature of the excited states using the $\Phi_{\mathrm{S}}$ metric that quantifies the overlap between the hole and electron densities as obtained in the attachment-detachment formalism (Figure S15, Table S4). ${ }^{36}$ Using this methodology, full charge-transfer (CT) [completely localized (LE)] excited states feature $\Phi_{\mathrm{S}}=0\left[\Phi_{\mathrm{S}}=1\right]$ can be inferred. $\Phi_{\mathrm{S}}$ values were calculated using the NANCY_EX package ${ }^{37}$ and the hole and the electron densities were visualised with DrawMol. ${ }^{38}$ Very low values, $\Phi_{\mathrm{S}}<0.12$ (Table S4), were calculated for both the $S_{1}$ and $T_{1}$ states for all three emitters, implying excited states with strong CT character. This is mirrored by the very small calculated oscillator strengths ranging from 0 to $10^{-4}$ and translates into radiative decay rates of $\sim 4.8 \times 10^{4} \mathrm{~s}^{-1}$ roughly two orders of magnitude smaller than the experimental values (Table S4). A range of other functionals were applied to SFX-PODPA-Me, with oscillator strength remaining very low (Table S6). Optimization of the singlet excited state was also performed for SFX-PO-DPA-Me and offered a slight increase in the predicted oscillator strength with a value of $2 \times 10^{-4}$ resulting in a radiative rate of $9.7 \times 10^{4} \mathrm{~s}^{-1}$, still much lower than the experimental value.

The discrepancy between the theoretical and the experimental radiative decay rates for the ground state and $\mathrm{S}_{1}$ equilibrium geometries of SFX-PO-DPA-Me suggests that the Franck-Condon approximation breaks down. Experimental and simulated emission are thus reconciled by invoking Herzberg-Teller intensity borrowing, mediated via vibronic coupling. Within this framework, perturbative interactions between the $\mathrm{S}_{1}$ electronic wave function and nuclear motions result in a combined electronic and vibronic state that can mix with nearby purely electronic states. We thus sum, consistently with the Thomas-Kuhn-Reiche sum-rules, the oscillator strengths associated with each 
vibronic transition from the ground state of vibration of state $S_{l}$ to the $n$-th vibrational levels of the ground state, $\mathrm{S}_{0}$. Considering the undistorted-undisplaced harmonic oscillator approximation and the same energy for all vibronic transitions $\Delta E_{S_{1} S_{0}}$ corresponding to the transition energy from the $S_{1}$ optimized geometry to the ground state, the oscillator strength for the transition between the $\mathrm{S}_{1}$ and the ground states is (see Supporting Information for further details):

$$
\begin{aligned}
& f^{S_{1} S_{0}}=\sum_{n} \frac{2}{3} \frac{m_{e}}{e^{2} \hbar^{2}} \Delta E_{S_{1} S_{0}} \sum_{\alpha=x, y, z}\left[\left\langle S_{1}, 0\left|\hat{\mu}_{\alpha}\right| S_{0}, n\right\rangle\right]^{2} \\
& =\frac{2}{3} \frac{m_{e}}{e^{2} \hbar^{2}} \Delta E_{S_{1} S_{0}} \sum_{\alpha=x, y, z}\left[\mu_{\alpha}^{S_{1} S_{0}, 0}\right]^{2}+\frac{2}{3} \frac{m_{e}}{e^{2} \hbar^{2}} \Delta E_{S_{1} S_{0}} \sum_{i} \sum_{\alpha=x, y, z}\left[\left(\frac{\partial \mu_{\alpha}^{S_{1} S_{0}}}{\partial Q_{i}}\right)_{0} \sqrt{\frac{\hbar}{2 \omega_{i}}}\right]^{2} \\
& =f_{0}+\sum_{i} f_{i}
\end{aligned}
$$

Where $\mathrm{m}_{\mathrm{e}}$ and $\mathrm{e}$ are the electron mass and charge, $\hbar$ is the reduced Planck constant, $\left\langle S_{1}, 0\left|\hat{\mu}_{\alpha}\right| S_{0}, n\right\rangle$ is the transition dipole moment matrix element between the ground state of vibration of $\mathrm{S}_{1}$ and the $n$-th vibrational level of $\mathrm{S}_{0}, \mu_{\alpha}^{S_{1} S_{0}, 0}$ is the $\alpha \equiv\{x, y, z\}$ component of the transition dipole moment computed at the $\mathrm{S}_{1}$ optimized geometry, $\left(\frac{\partial \mu_{\alpha}^{S_{1} S_{0}}}{\partial Q_{i}}\right)$ is the first derivative of the electronic transition dipole moment along the normal coordinates $Q_{i}$ evaluated at the equilibrium geometry of the reference $\mathrm{S}_{1}$ state, $\omega_{i}$ is the angular frequency of the $i$ th normal mode; $f_{0}$ and $f_{i}$ are the oscillator strengths computed at the $\mathrm{S}_{1}$ optimized geometry and the Herzberg-Teller contribution of the i-th normal mode, respectively. 


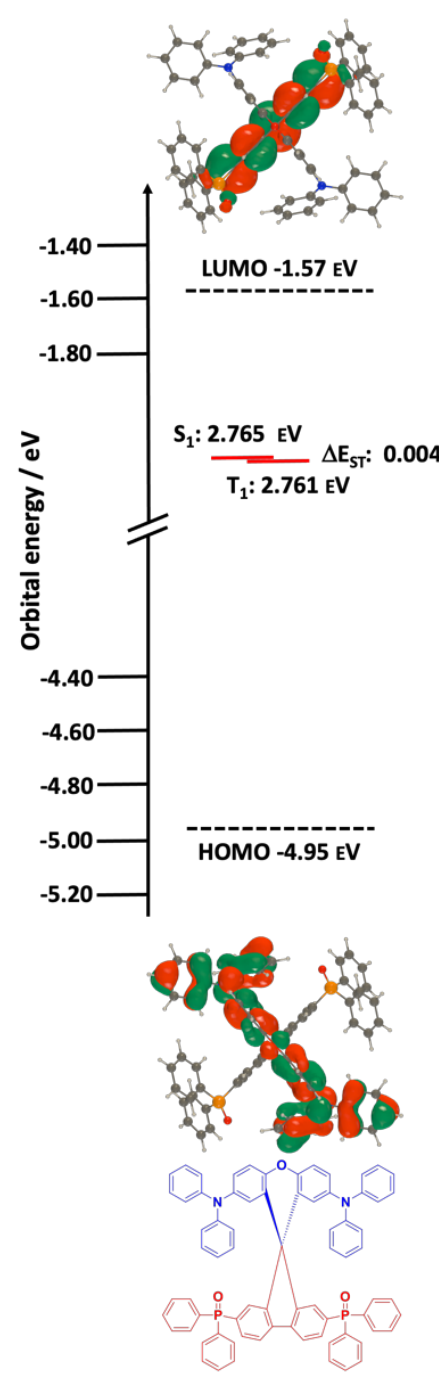

SFX-PO-DPA

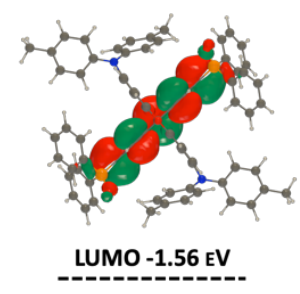

$\frac{\mathrm{SV}_{1}: 2.651 \mathrm{EV}}{\overline{\mathrm{T}_{1}: 2.648 \mathrm{EV}}} \Delta E_{\mathrm{sT}}: 0.004 \mathrm{EV}$

$\frac{\mathrm{S}_{1}: 2.343 \mathrm{EV}}{\mathrm{T}_{1}: 2.338 \mathrm{EV}} \Delta E_{\mathrm{ST}}: 0.005 \mathrm{EV}$

HOMO $-4.49 \mathrm{EV}$

HOMO $-4.81 \mathrm{EV}$
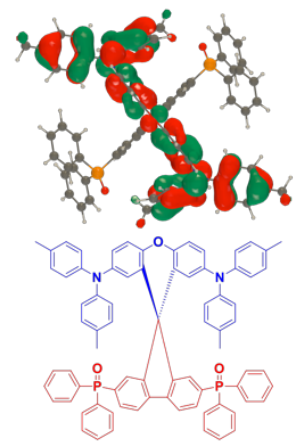

SFX-PO-DPA-Me

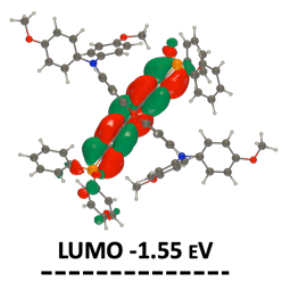

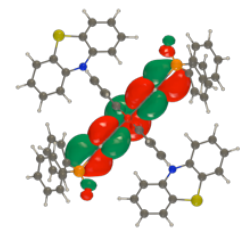
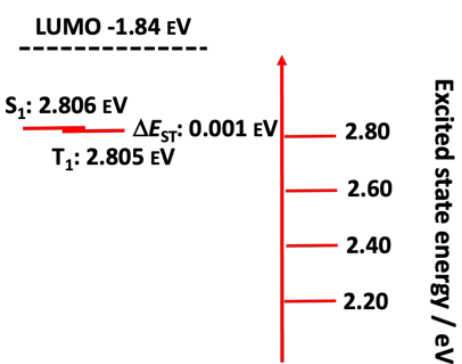

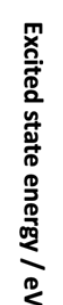

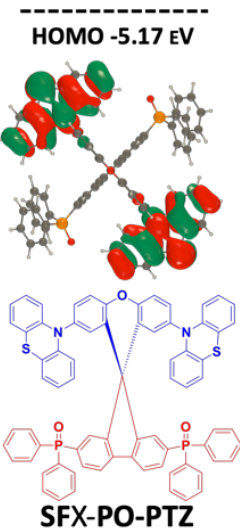

Figure 9. Calculated HOMO, LUMO (as obtained at DFT PBE0 6-31G(d,p) level) $\mathrm{S}_{1}$ and $\mathrm{T}_{1}$ energies (as obtained at TDA-DFT PBE0 6-31G(d,p) level), and contour plots of the HOMO and LUMO orbitals of SFX-PO-DPA, SFX-PO-DPA-Me, SFX-PO-DPA-OMe and SFX-PO-PTZ.

In practice, the transition dipole moment derivative $\left(\frac{\partial \mu_{\alpha}^{S_{1} S_{0}}}{\partial Q_{i}}\right)_{0}$ is obtained by displacing the $\mathrm{S}_{1-}$ optimized excited-state geometry along its normal modes by $0.01 \AA \sqrt{a m u}$ in an interval going from 0.1 to $0.1 \AA \sqrt{a m u}$ and fitting the transition dipole moment evolution as a function of the normal coordinate, $Q_{i}$, with a linear regression (see Table S7). To reduce computational cost, we selected vibrational modes leading to a concomitant bending of the electron-donating and electron-accepting 
units, as these sensibly increase the hole and electron densities near the $\mathrm{sp}^{3}$ hybridized carbon and therefore could prompt larger oscillator strength (Figure 10 S16, Table S8). Accounting for these vibrations indeed results in an increase in oscillator strength up to $3.7 \times 10^{-4}$. This now brings the calculated radiative decay rate (considering a refractive index of 1.73 for $\mathrm{mCP}$ ) to $1.81 \times 10^{5} \mathrm{~s}^{-1}$, which is within the same order of magnitude as the measured value of $k_{\mathrm{r}}=2.3 \times 10^{6} \mathrm{~s}^{-1}$, highlighting the importance of Herzberg-Teller effects in spiro-based compounds. We expect the (dynamic, i.e. induced by vibrations) increase in electron-hole overlap and in the ensuing radiative decay rate to be sensitive to the nature of the electron-active substituents. To verify this hypothesis, we designed compound SFXPO-PTZ, where the DPA-Me side groups are replaced by stronger electron-donating phenothiazine (PTZ) groups, and carried out the same analysis, i.e., $\mathrm{S}_{1}$ excited-state optimization followed by sampling along the relevant normal modes (Table S9). As expected, SFX-PO-PTZ exhibits an oscillator strength in the relaxed $\mathrm{S}_{1}$ excited-state geometry that amounts to $7.13 \times 10^{-5}$ when HerzbergTeller corrections are included, which is one order of magnitude smaller than SFX-PO-DPA-Me. This result can be readily explained by the removal of the hole density away from the $\mathrm{sp}^{3}$ carbon in presence of the stronger PTZ donor moieties (Figure S17, Table S8).

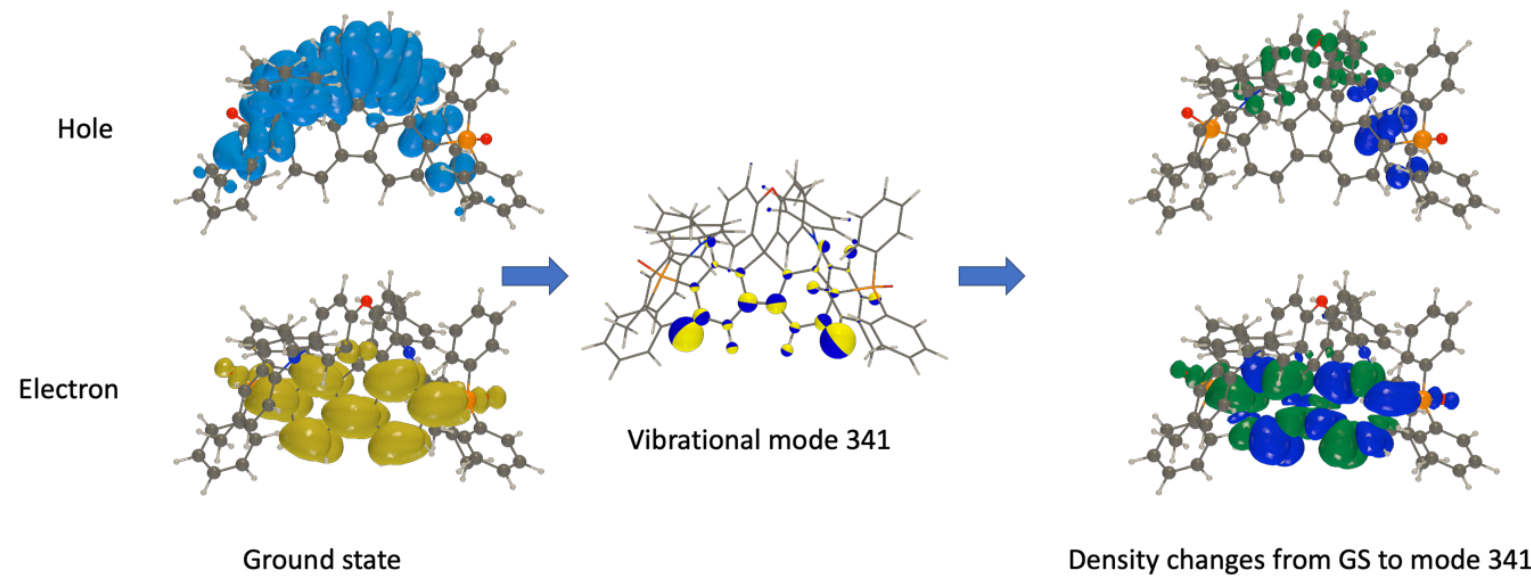

Figure 10. Hole $\left(\mathrm{h}^{+}\right)$electron $\left(\mathrm{e}^{-}\right)$densities of SFX-PO-DPA-OMe for the undistorted (left panel) and distorted (right panel) $\mathrm{S}_{1}$ along the vibrational mode at $1556.2 \mathrm{~cm}^{-1}$ (centre panel) leading to the largest enhancement in oscillator strength. The size and the orientation of the yellow and blue refer to the 
amplitude and the displacement vector, respectively. Difference hole and electron densities between the distorted $(0.1 \AA \sqrt{a m u})$ and the undistorted geometry with the green color for positive lobes and blue for negative ones (right panel). The isovalue for the hole and electron densities amounts to $4 \times 10^{-4}$ while for the difference hole and electron densities, it amounts to $4 \times 10^{5}$.

\section{Device Fabrication.}

The photophysical picture points to spiro-based compounds that are TADF emitters and have high $\Phi_{\mathrm{PL}}$ in $\mathrm{mCP}$ and short $\tau_{\mathrm{d}}$. These compounds were therefore assessed as emitters in OLEDs. The OLED device stack architecture is shown in Figure 11 and consists of: ITO / NPB (30 nm) / TCTA (20 $\mathrm{nm}) / \mathrm{mCP}(10 \mathrm{~nm}) /$ Emitter: mCP (20 nm) / DPEPO (10 nm) / TmPyPB (40 nm) / LiF (1 nm) / Al (100 nm), where $N, N^{\prime}$-bis(naphthalen-1-yl)- $N, N^{\prime}$-bis(phenyl)benzidine (NPB) was used as a hole injection layer (HIL), tris(4-carbazoyl-9-ylphenyl)amine (TCTA) was used as a hole transporting layer (HTL), mCP (1,3-bis( $N$-carbazolyl)benzene) and DPEPO were used as an electron and hole blockers, respectively. 1,3,5-tris(3-pyridyl-3-phenyl)benzene (TmPyPB) was chosen as the electron transporting layer (ETL) as it possesses a high electron mobility of $10^{-4} \mathrm{~cm}^{2} \mathrm{~V}^{-1} \mathrm{~s}^{-1}$ and a high triplet energy of 2.75 $\mathrm{eV}$ along with a deep HOMO energy of $6.7 \mathrm{eV} .{ }^{39}$ The emissive layer (EML) consisted of $10 \mathrm{wt} \%$ doped films of SFX-PO-DPA, SFX-PO-DPA-OMe and $15 \mathrm{wt} \%$ doped film of SFX-PO-DPA-Me in mCP.

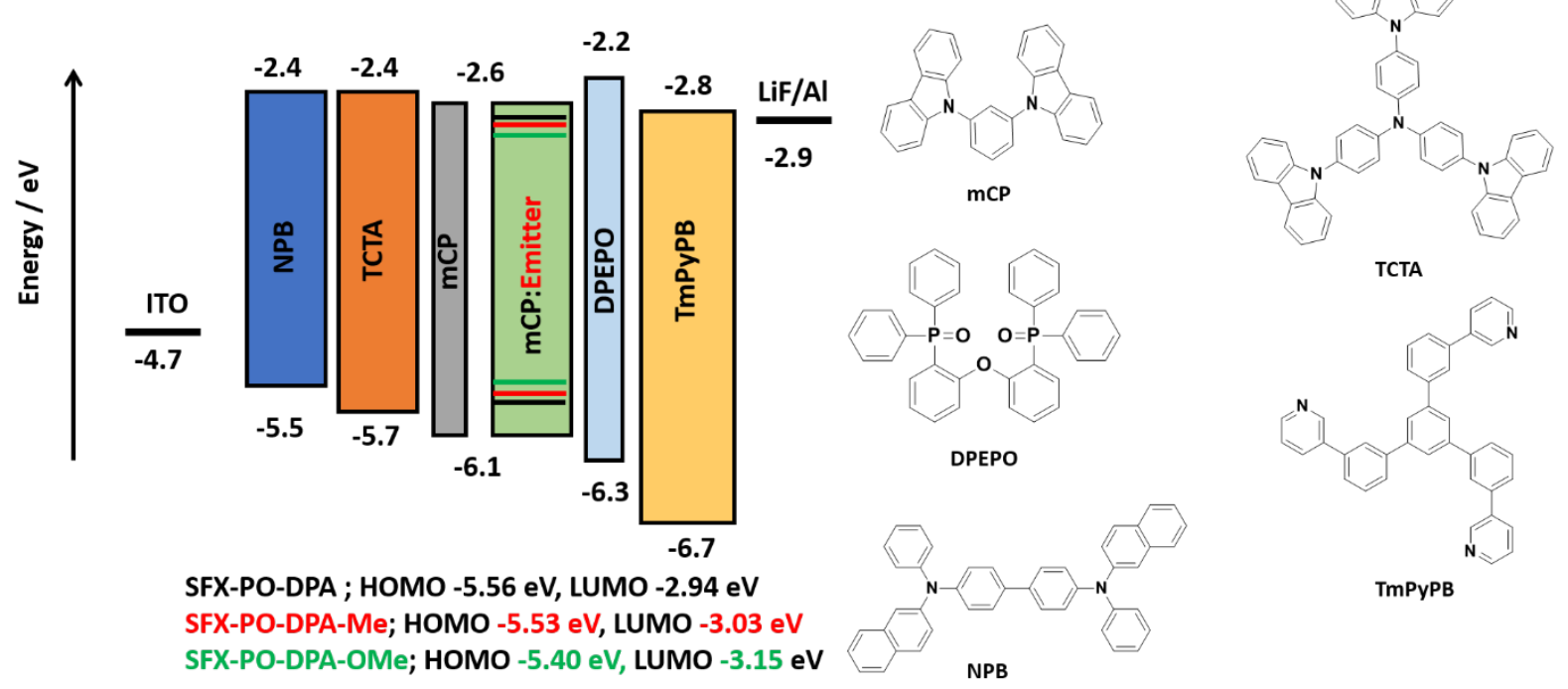

Figure 11. Chemical structures and energy levels of materials used for device fabrication. 
The electroluminescence properties are shown in Figure $\mathbf{1 2}$ and data are summarized in Table 3. The three emitters exhibited blue-green to green electroluminescence with systematic red-shifting of the EL spectra as a function of donor strength, in parallel to the trends and maxima observed in their respective PL spectra. The OLED with SFX-PO-DPA showed a $\lambda_{\mathrm{EL}}$ of $500 \mathrm{~nm}$ with CIE coordinates of $(0.20,0.47)$, the OLED with SFX-PO-DPA-Me showed a slight red-shift with a $\lambda_{\mathrm{EL}}$ of $502 \mathrm{~nm}$ and CIE coordinates of $(0.23,0.50)$ and SFX-PO-DPA-OMe displayed the most red-shifted EL spectrum with a $\lambda_{\mathrm{EL}}$ of $520 \mathrm{~nm}$ and CIE coordinates of $(0.29,0.56)$. All OLEDs showed steep current-voltageluminance behavior (Figures 12a and 12b) with low turn-on voltages of $3.2 \mathrm{~V}$ for devices within SFXPO-DPA and SFX-PO-DPA-Me and 3.8 V for the device with SFX-PO-DPA-OMe.

(a)

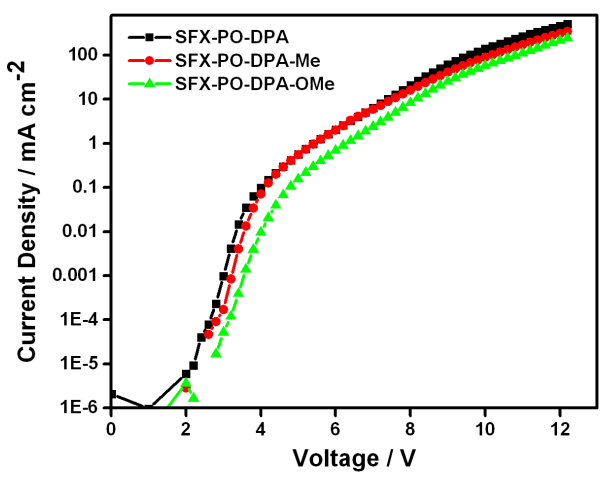

(c)

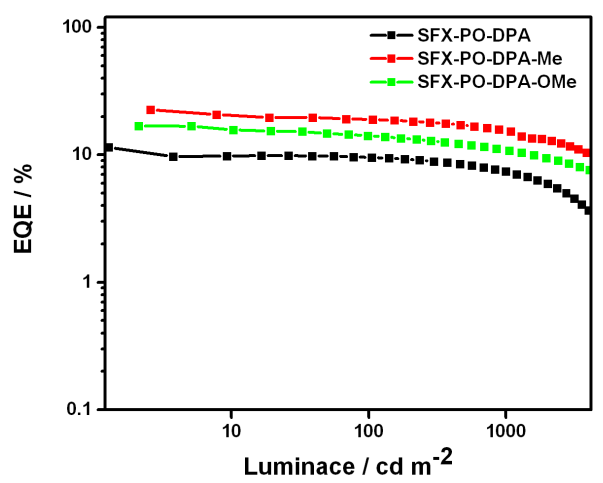

(b)

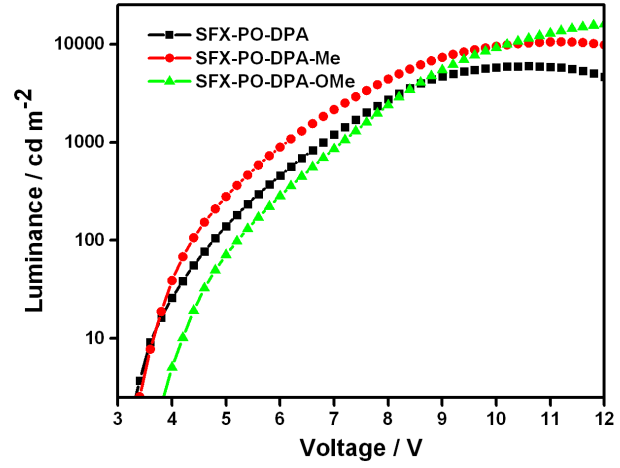

(d)

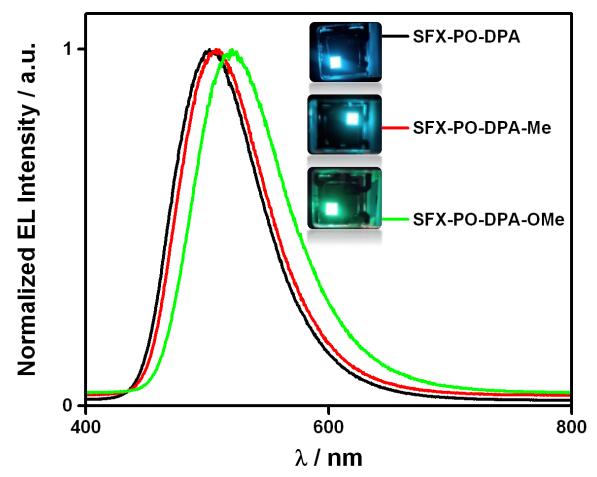

Figure 12 (a) Current density-voltage characteristics (b) Luminance vs Voltage. (c) EQE vs Luminance (d) Normalized EL spectra of SFX-PO-DPA (black), SFX-PO-DPA-Me (red) and SFX-PO-DPAOMe (green). 
Figure 12c shows the EQE vs luminance behavior of the three devices. The OLED with SFXPO-DPA-Me showed an excellent device performance with an $\mathrm{EQE}_{\max }$ of $23 \%$ at a brightness of 2.5 $\mathrm{cd} \mathrm{m}^{-2}$ and relatively low roll-off, with an $\mathrm{EQE}_{100}$ of $19 \%$ at a display-relevant brightness of $100 \mathrm{~cd} \mathrm{~m}^{-}$ ${ }^{2}$. Similarly, the OLEDs with SFX-PO-DPA and SFX-PO-DPA-OMe also maintained high EQE max values of $11 \%$ and $16 \%$, respectively, at low luminance, and likewise showed low efficiency roll-off with $\mathrm{EQE}_{100}$ of $9 \%$ and $15 \%$, respectively. Even at a luminance of $1000 \mathrm{~cd} \mathrm{~m}^{-2}, \mathrm{EQE}_{1000}$ of $7 \%, 16 \%$ and 12\% were maintained for the OLEDs with SFX-PO-DPA, SFX-PO-DPA-Me and SFX-PO-DPAOMe, respectively. The high observed EQEs are indicative of efficient triplet harvesting while the low efficiency roll-offs are due to the short $\tau_{\mathrm{d}}$ values, which are beneficial in reducing the various triplet exciton quenching mechanisms such as triplet-triplet annihilation (TTA) and triplet polaron annihilation (TPA) in the devices. The poorer device performance of the OLEDs with SFX-PO-DPA and SFX-PODPA-OMe can be correlated to their lower $\Phi_{\mathrm{PL}}$ values (Table 1). Compared to the reported OLEDs with spiro-based emitters shown in Figure 1, the devices based on SFX-PO-DPA-Me show the best performance in terms of $\mathrm{EQE}_{\max }$ and reduced efficiency roll-off. Further, SFX-PO-DPA and SFX-PODPA-OMe emitters show comparable $\mathrm{EQE}_{\max }$ values and improved efficiency roll-off characteristics compared to literature devices.

Table 3. Electroluminescence Properties of SFX-PO-DPA, SFX-PO-DPA-Me and SFX-PO-DPAOMe.

\begin{tabular}{|c|c|c|c|c|c|c|}
\hline & $\begin{array}{l}\mathbf{V}_{\text {on }}{ }^{\mathbf{a}} \\
/ \mathbf{V}\end{array}$ & $\begin{array}{l}\lambda_{\mathbf{E L}} \mathbf{b} \\
/ \mathbf{n m}\end{array}$ & $\begin{array}{c}\mathrm{EQE}_{\max }^{\mathrm{c}} \\
\mathrm{EQE}_{100}{ }^{\mathrm{d}} ; \mathrm{EQE}_{1000}^{\mathrm{e}} \\
/ \%\end{array}$ & $\begin{array}{l}\mathrm{CE}_{\max }^{\mathrm{c}} \\
/ \mathbf{c d ~ A}^{-1}\end{array}$ & $\begin{array}{l}\mathbf{P E}_{\max }^{\mathrm{c}} \\
/ \operatorname{lm} \mathbf{W}^{-1}\end{array}$ & $\begin{array}{l}\text { CIE }^{\mathbf{f}} \\
/(\mathbf{x}, \mathbf{y})\end{array}$ \\
\hline SFX-PO-DPA & 3.2 & 490 & $11 ; 9 ; 7$ & 30.6 & 29.7 & $(0.21,0.47)$ \\
\hline SFX-PO-DPA-Me & 3.2 & 500 & $23 ; 19 ; 16$ & 62.8 & 58.1 & $(0.23,0.50)$ \\
\hline SFX-PO-DPA-OMe & 3.8 & 520 & $16 ; 14 ; 12$ & 53.1 & 43.9 & $(0.29,0.56)$ \\
\hline \multicolumn{7}{|c|}{$\begin{array}{l}{ }^{\text {a. }} \text { Measured at } 1 \mathrm{~cd} / \mathrm{m}^{2} .{ }^{\text {b. }} \text { Emission maximum at } 1 \mathrm{~mA} / \mathrm{cm}^{2} .{ }^{c .} \text { Maximum efficie } \\
\mathrm{cd} / \mathrm{m}^{2} \text {. } \quad{ }^{\mathrm{f} .} \text { Commission Internationale de l'Éclairage coordinates at } 1 \mathrm{~mA} / \mathrm{cm}^{2} \text {. }\end{array}$} \\
\hline
\end{tabular}




\section{Conclusions.}

We have successfully synthesized a series of spiro-configured xanthene-based TADF emitters, which exhibit a confluence of desirable photophysical properties. We have demonstrated an efficient synthetic strategy to incorporate SFX bridge to realize highly sterically demanding structures that translate into materials that possess short delayed lifetimes of $<10 \mu \mathrm{s}$, very small $\Delta \mathrm{E}_{\text {ST }}$ values coupled with high $\Phi_{\mathrm{PL}}$ in the solid state. Molecular modelling reveals that the emission in these compounds occurs through a Herzberg-Teller mechanism where some intramolecular vibrations promote larger overlapping electron and hole density in vicinity of the $s p^{3}$ carbon atoms, thereby enhancing the radiative decay rate. The designed compounds exhibit non-radiative decay rates an order of magnitude slower than the rate of reverse intersystem crossing. The resulting OLEDs exhibited EQE $E_{\max }$ values as high as $23 \%$ and with only modest efficiency roll-off at 100 and $1000 \mathrm{~cd} \mathrm{~m}^{-2}$ as a result of the short delayed lifetimes and reduced triplet-triplet and triplet-polaron annihilation associated with the expected reduced triplet diffusion due to the bulky shape of our compounds. These results clearly illustrated how crucial an SFX blocking unit is in realizing highly efficient TADF systems, which we believe will reinvigorate the importance of spiro system in TADF community.

\section{Supporting Information}

Synthesis protocols, NMR spectra, crystallographic data, supplementary photophysical measurements, computational data obtained from DFT and TD DFT and electroluminescence data.

\section{Acknowledgements}

E.Z.-C. thanks the Leverhulme Trust (No. RPG-2016-047) and the University of St Andrews for support. We are grateful to the EPSRC for financial support (grants EP/P007805/1, EP/ P010482/1, EP/L017008/1, EP/J01771X, and EP/J00916). M.R.M. thanks the Innovation Programme H2020MSCA-IF-2014-659237 for financial support. The authors thank Dr. Gary Nichol for the 
crystallographic data collection and refinement; The University of Edinburgh for funding the diffractometer purchase. W.L. thanks the China Scholarship Council (grant number 201708060003). V. L. thanks the F.R.S.-FNRS for his Research Associate position. Computational resources have been provided by the Consortium des Équipements de Calcul Intensif (CÉCI), funded by the Fonds de la Recherche Scientifiques de Belgique (F.R.S.-FNRS) under Grant No. 2.5020.11, GEQ U.G006.15, 1610468, and RW/GEQ2016). DB is a FNRS Research Director. Y.O. acknowledges fruitful discussions with Prof. Juan-Carlos Sancho-Garcia from the University of Alicante and Prof. Luca Muccioli from the University of Bologna.

\section{References}

(1) Yang, Z.; Mao, Z.; Xie, Z.; Zhang, Y.; Liu, S.; Zhao, J.; Xu, J.; Chi, Z.; Aldred, M. P., Recent advances in organic thermally activated delayed fluorescence materials. Chem. Soc. Rev. 2017, 46, 915-1016.

(2) Liu, Y.; Li, C.; Ren, Z.; Yan, S.; Bryce, M. R., All-organic thermally activated delayed fluorescence materials for organic light-emitting diodes. Nat. Rev. Mater. 2018, 3, 18020.

(3) Wong, M. Y.; Zysman-Colman, E., Purely Organic Thermally Activated Delayed Fluorescence Materials for Organic Light-Emitting Diodes. Adv Mater 2017, 29, 1605444.

(4) Cai, X.; Su, S.-J., Marching Toward Highly Efficient, Pure-Blue, and Stable Thermally Activated Delayed Fluorescent Organic Light-Emitting Diodes. Adv. Funct. Mater. 2018, 28.

(5) Zhang, Q.; Li, B.; Huang, S.; Nomura, H.; Tanaka, H.; Adachi, C., Efficient blue organic light-emitting diodes employing thermally activated delayed fluorescence. Nat. Photonics 2014, 8, 326-332.

(6) Hirata, S.; Sakai, Y.; Masui, K.; Tanaka, H.; Lee, S. Y.; Nomura, H.; Nakamura, N.; Yasumatsu, M.; Nakanotani, H.; Zhang, Q.; Shizu, K.; Miyazaki, H.; Adachi, C., Highly efficient blue electroluminescence based on thermally activated delayed fluorescence. Nat Mater 2015, 14, 330-336.

(7) Kim, G. H.; Lampande, R.; Im, J. B.; Lee, J. M.; Lee, J. Y.; Kwon, J. H., Controlling the exciton lifetime of blue thermally activated delayed fluorescence emitters using a heteroatom-containing pyridoindole donor moiety. Materials Horizons 2017, 4, 619-624.

(8) Sun, J. W.; Baek, J. Y.; Kim, K.-H.; Moon, C.-K.; Lee, J.-H.; Kwon, S.-K.; Kim, Y.-H.; Kim, J.-J., Thermally Activated Delayed Fluorescence from Azasiline Based Intramolecular Charge-Transfer Emitter (DTPDDA) and a Highly Efficient Blue Light Emitting Diode. Chem. Mater. 2015, 27, 6675-6681.

(9) Lin, T.-A.; Chatterjee, T.; Tsai, W.-L.; Lee, W.-K.; Wu, M.-J.; Jiao, M.; Pan, K.-C.; Yi, C.-L.; Chung, C.L.; Wong, K.-T.; Wu, C.-C., Sky-Blue Organic Light Emitting Diode with 37\% External Quantum Efficiency Using Thermally Activated Delayed Fluorescence from Spiroacridine-Triazine Hybrid. Adv. Mater. 2016, 28, 6976-6983.

(10) Saragi, T. P. I.; Spehr, T.; Siebert, A.; Fuhrmann-Lieker, T.; Salbeck, J., Spiro Compounds for Organic Optoelectronics. Chem. Rev. 2007, 107, 1011-1065.

(11) Luo, J.; Zhou, Y.; Niu, Z.-Q.; Zhou, Q.-F.; Ma, Y.; Pei, J., Three-Dimensional Architectures for Highly Stable Pure Blue Emission. J. Am. Chem. Soc. 2007, 129, 11314-11315.

(12) Gu, J.-F.; Xie, G.-H.; Zhang, L.; Chen, S.-F.; Lin, Z.-Q.; Zhang, Z.-S.; Zhao, J.-F.; Xie, L.-H.; Tang, C.; Zhao, Y.; Liu, S.-Y.; Huang, W., Dumbbell-Shaped Spirocyclic Aromatic Hydrocarbon to Control 
Intermolecular $\pi-\pi$ Stacking Interaction for High-Performance Nondoped Deep-Blue Organic LightEmitting Devices. The Journal of Physical Chemistry Letters 2010, 1, 2849-2853.

(13) Seo, J.-A.; Gong, M. S.; Lee, J. Y., Thermally stable indoloacridine type host material for high efficiency blue phosphorescent organic light-emitting diodes. Org. Electron. 2014, 15, 3773-3779.

(14) Poriel, C.; Sicard, L.; Rault-Berthelot, J., New generations of spirobifluorene regioisomers for organic electronics: tuning electronic properties with the substitution pattern. Chem. Commun. 2019, 55, 14238-14254.

(15) Lucas, F.; Ibraikulov, O. A.; Quinton, C.; Sicard, L.; Heiser, T.; Tondelier, D.; Geffroy, B.; Leclerc, N.; Rault-Berthelot, J.; Poriel, C., Spirophenylacridine-2,7-(diphenylphosphineoxide)-fluorene: A Bipolar Host for High-Efficiency Single-Layer Blue Phosphorescent Organic Light-Emitting Diodes. Adv. Optical Mater. 2020, 8, 1901225.

(16) Nakagawa, T.; Ku, S. Y.; Wong, K. T.; Adachi, C., Electroluminescence based on thermally activated delayed fluorescence generated by a spirobifluorene donor-acceptor structure. Chem. Commun. 2012, 48, 9580-9582.

(17) Mehes, G.; Nomura, H.; Zhang, Q.; Nakagawa, T.; Adachi, C., Enhanced electroluminescence efficiency in a spiro-acridine derivative through thermally activated delayed fluorescence. Angew Chem Int Ed Eng/ 2012, 51, 11311-11315.

(18) Nasu, K.; Nakagawa, T.; Nomura, H.; Lin, C. J.; Cheng, C. H.; Tseng, M. R.; Yasuda, T.; Adachi, C., A highly luminescent spiro-anthracenone-based organic light-emitting diode exhibiting thermally activated delayed fluorescence. Chem. Commun. 2013, 49, 10385-10387.

(19) Ohkuma, H.; Nakagawa, T.; Shizu, K.; Yasuda, T.; Adachi, C., Thermally Activated Delayed Fluorescence from a Spiro-diazafluorene Derivative. Chem. Lett. 2014, 43, 1017-1019.

(20) Wang, Y.-K.; Wu, S.-F.; Yuan, Y.; Li, S.-H.; Fung, M.-K.; Liao, L.-S.; Jiang, Z.-Q., Donor- $\sigma-A c c e p t o r$ Molecules for Green Thermally Activated Delayed Fluorescence by Spatially Approaching Spiro Conformation. Org. Lett. 2017, 19, 3155-3158.

(21) Rao, J.; Zhao, C.; Wang, Y.; Bai, K.; Wang, S.; Ding, J.; Wang, L., Achieving Deep-Blue Thermally Activated Delayed Fluorescence in Nondoped Organic Light-Emitting Diodes through a Spiro-Blocking Strategy. ACS Omega 2019, 4, 1861-1867.

(22) Zhu, X.-D.; Peng, C.-C.; Kong, F.-C.; Yang, S.-Y.; Li, H.-C.; Kumar, S.; Wang, T.-T.; Jiang, Z.-Q.; Liao, L.-S., Acceptor modulation for improving a spiro-type thermally activated delayed fluorescence emitter. J. Mater. Chem. C 2020, 8, 8579-8584.

(23) Wang, Y.-K.; Huang, C.-C.; Ye, H.; Zhong, C.; Khan, A.; Yang, S.-Y.; Fung, M.-K.; Jiang, Z.-Q.; Adachi, C.; Liao, L.-S., Through Space Charge Transfer for Efficient Sky-Blue Thermally Activated Delayed Fluorescence (TADF) Emitter with Unconjugated Connection. Adv. Optical Mater. 2020, 8, 1901150.

(24) Poriel, C.; Cocherel, N.; Rault-Berthelot, J.; Vignau, L.; Jeannin, O., Incorporation of spiroxanthene units in blue-emitting oligophenylene frameworks: a new molecular design for OLED applications. Chemistry 2011, 17, 12631-12645.

(25) Xie, L.-H.; Liu, F.; Tang, C.; Hou, X.-Y.; Hua, Y.-R.; Fan, Q.-L.; Huang, W., Unexpected One-Pot Method to Synthesize Spiro[fluorene-9, $9^{\prime}$-xanthene] Building Blocks for Blue-Light-Emitting Materials. Org. Lett. 2006, 8, 2787-2790.

(26) Maciejczyk, M.; Ivaturi, A.; Robertson, N., SFX as a low-cost 'Spiro' hole-transport material for efficient perovskite solar cells. J. Mater. Chem. A 2016, 4, 4855-4863.

(27) Zhao, J.; Xie, G.-H.; Yin, C.-R.; Xie, L.-H.; Han, C.-M.; Chen, R.-F.; Xu, H.; Yi, M.-D.; Deng, Z.-P.; Chen, S.-F.; Zhao, Y.; Liu, S.-Y.; Huang, W., Harmonizing Triplet Level and Ambipolar Characteristics of WideGap Phosphine Oxide Hosts toward Highly Efficient and Low Driving Voltage Blue and Green PHOLEDs: An Effective Strategy Based on Spiro-Systems. Chem. Mater. 2011, 23, 5331-5339.

(28) Sadighi, J. P.; Harris, M. C.; Buchwald, S. L., A highly active palladium catalyst system for the arylation of anilines. Tetrahedron Lett. 1998, 39, 5327-5330.

(29) Fungo, F.; Wong, K.-T.; Ku, S.-Y.; Hung, Y.-Y.; Bard, A. J., Electrogenerated Chemiluminescence. 81. Influence of Donor and Acceptor Substituents on the ECL of a Spirobifluorene-Bridged Bipolar System. J. Phys. Chem. B 2005, 109, 3984-3989. 
(30) Connelly, N. G.; Geiger, W. E., Chemical Redox Agents for Organometallic Chemistry. Chem. Rev. 1996, 96, 877-910.

(31) Cardona, C. M.; Li, W.; Kaifer, A. E.; Stockdale, D.; Bazan, G. C., Electrochemical Considerations for Determining Absolute Frontier Orbital Energy Levels of Conjugated Polymers for Solar Cell Applications. Adv. Mater. 2011, 23, 2367-2371.

(32) Masui, K.; Nakanotani, H.; Adachi, C., Analysis of exciton annihilation in high-efficiency sky-blue organic light-emitting diodes with thermally activated delayed fluorescence. Org. Electron. 2013, 14, 2721-2726.

(33) Chen, Z.; Wu, Z.; Ni, F.; Zhong, C.; Zeng, W.; Wei, D.; An, K.; Ma, D.; Yang, C., Emitters with a pyridine-3,5-dicarbonitrile core and short delayed fluorescence lifetimes of about $1.5 \mu \mathrm{s}$ : orange-red TADF-based OLEDs with very slow efficiency roll-offs at high luminance. J. Mater. Chem. C 2018, 6, 6543-6548.

(34) Melhuish, W. H., Quantum Efficiences Of Fluorescence Of Organic Substances: Effect Of Solvent And Concentration Of The Fluorescent Solute 1. J. Phys. Chem. 1961, 65, 229-235.

(35) Moral, M.; Muccioli, L.; Son, W. J.; Olivier, Y.; Sancho-García, J. C., Theoretical Rationalization of the Singlet-Triplet Gap in OLEDs Materials: Impact of Charge-Transfer Character. J. Chem. Theory Comput. 2015, 11, 168-177.

(36) Olivier, Y.; Moral, M.; Muccioli, L.; Sancho-García, J.-C., Dynamic nature of excited states of donor-acceptor TADF materials for OLEDs: how theory can reveal structure-property relationships. J. Mater. Chem. C 2017, 5, 5718-5729.

(37) Etienne, T.; Assfeld, X.; Monari, A., Toward a Quantitative Assessment of Electronic Transitions' Charge-Transfer Character. J. Chem. Theory Comput. 2014, 10, 3896-3905.

(38) V. Liégeois, DrawMol, UNamur, https://www.unamur.be/drawmol, 2021.

(39) Su, S.-J.; Chiba, T.; Takeda, T.; Kido, J., Pyridine-Containing Triphenylbenzene Derivatives with High Electron Mobility for Highly Efficient Phosphorescent OLEDs. Adv. Mater. 2008, 20, 2125-2130.

(40) Grisanti, L.; Olivier, Y.; Wang, L.; Athanasopoulos, S.; Cornil, J.; Beljonne, D., Roles of local and nonlocal electron-phonon couplings in triplet exciton diffusion in the anthracene crystal. Physical Review B 2013, 88, 035450.

\section{TOC graphic}

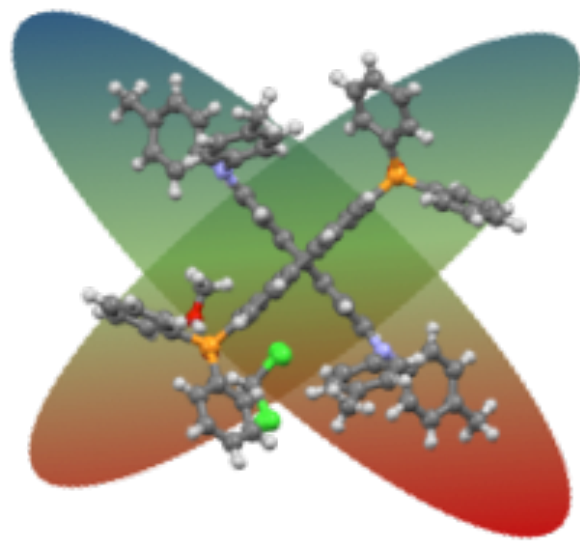

\section{$\Delta E_{\mathrm{ST}} \sim 0, \Phi_{\mathrm{PL}}>70 \%, \tau_{\mathrm{d}}$} $<10 \mu \mathrm{s}, \mathrm{EQE}_{\max }>20 \%$

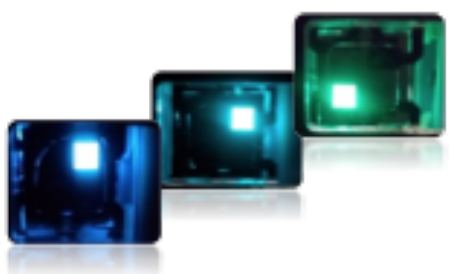

